\title{
Effects of jet angle on harmonic structure of sound radiating from the flute
}

\author{
Kimie Onogi*, Hiroshi Yokoyama, and Akiyoshi Iida \\ Department of Mechanical Engineering, Toyohashi University of Technology, 441-8580 Aichi, Japan
}

Received 27 May 2020, Accepted 16 January 2021

\begin{abstract}
For an isolated flute head joint, the effects of jet angle on harmonic structure of a single note are investigated within the practical range for human players. The mechanisms of these effects are discussed on the basis of both the radiated sound and the flow field measured with a hot-wire anemometer. The blowing parameters, viz., jet angle (angle between jet direction and window), jet offset (relative height of jet direction from the edge), lip-to-edge distance, and flow rate, were varied independently by using an artificial blowing device based on measured conditions for a human player, where the jet direction is defined as that measured without the head joint. The radiated sound revealed that jet angle varied the differential sound pressure level of the second to third harmonic $(\Delta \mathrm{SPL})$ less than jet offset, however, as much as flow rate and more than lip-to-edge distance. The spatial distribution of jet fluctuation center showed that, with increasing jet angle (the jet direction approaches vertical to the window), the jet deflected more inside, so that the actual jet offset was estimated to be further inside. The variation of $\Delta \mathrm{SPL}$ with jet angle seems to be caused mainly by this shift in the actual jet offset.
\end{abstract}

Keywords: Flute, Harmonic structure, Jet angle, Jet offset

\section{Introduction}

The flute belongs to a family of flue instruments that also includes the recorder, organ pipe, shakuhachi and panpipes. When a player blows a flue instrument, an air-jet travels across an opening (window) in the resonator (pipe) toward a sharp edge (labium) (see Fig. 1a). During traveling, the jet amplifies spatially $[1,2]$ and fluctuates periodically in the vertical direction. This jet fluctuation delays from the oral cavity exit to the edge [3-5]. The pressure in the resonator also fluctuates periodically. If the jet reaches the edge and enters into the resonator when the pressure in the resonator becomes high, the jet promotes compression in the resonator, and the high pressure in the resonator is further intensified. This phase relation corresponds to the favorable relation for the acoustic power generation [6]. To form this phase relation, the time delay of the jet fluctuation is to be almost half a period [7-9]. Further, the acoustic fluctuations around the exit induce a modulation of the jet fluctuation as acoustic feedback. Then, the feedback loop with the period of resonant frequency is formed $[8,10]$ (see Fig. 1b).

Unlike the recorder and organ pipe, but like the shakuhachi and panpipes, sound radiating from the flute varies not only with instrument shape or flow rate but also with

*Corresponding author: onogi@aero.me.tut.ac.jp lip position relative to the instrument and lip shapes [11, 12]. Flutists develop the ability to control their lips and flow rate precisely in order to enhance the artistic value of the music played. Various investigations have been conducted on the physical conditions that govern the sound of the flute. Ando [11] has estimated the possible ranges of blowing parameters, including jet angle (angle between jet direction and the window), jet offset (relative height of jet direction from the edge), lip-to-edge distance, and flow rate, by using an artificial blowing device. In his subsequent study [12], the effects of parameters on timbre have been evaluated based on the difference of sound pressure levels between even and odd harmonics. The actual blowing conditions for lip-to-edge distance, shapes of cavity exit, and mouth pressure have been measured for flute players through photo and pressure measurements by de la Cuadra [13] and Vauthrin et al. [14]. Among these parameters, preliminary experiments by Ando [11] have indicated that the effect of jet angle on harmonic structure (sound pressure levels of harmonics), which affects timbre, is weaker than the effects of jet offset, lip-to-edge distance, and jet velocity; however, quantitative results have not yet been demonstrated.

The mechanisms whereby these parameters affect the radiated sound have been studied by various researchers. Fletcher and Douglas [7] and Yoshikawa [15] have presented the theoretical calculation for the variation of 

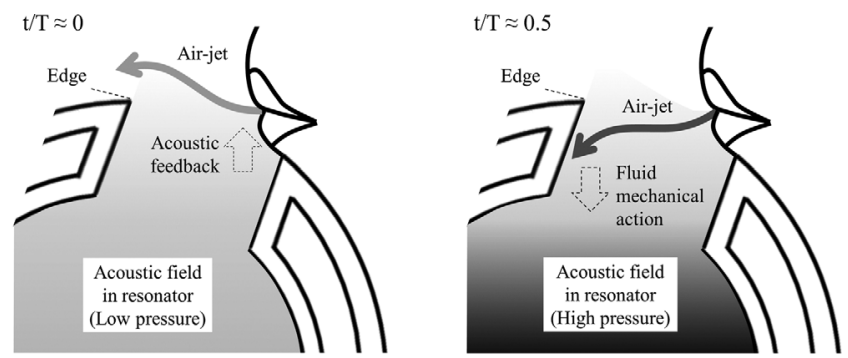

(a) Jet-acoustic field interaction

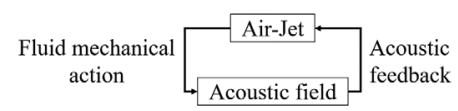

(b) Feedback loop

Figure 1. Feedback loop between jet and acoustic field.

harmonic structure with respect to jet offset in a simplified organ pipe model. Coltman [16] has predicted the variation by time domain simulations for the flute. These studies assume that the jet oscillates symmetrically with respect to the vertical center of the jet exit as it travels across the window. However, Verge et al. [17] have reviled a jet deflection in the initial transient in a recorder-like organ pipe with an artificial blowing device. Later, Auvray and Fabre [18] have conducted jet visualizations for panpipes and proposed a formula that describes a jet deflection during traveling. The jet deflection has also been observed in simulations of the recorder; thus, Onogi et al. [19] have proposed a formula for jet displacement that takes into account the jet deflection. These studies imply that the resulting jet offset is not predictable only from the geometrical conditions of the jet exit and edge. Further, lip-to-edge distance and jet velocity have been shown to affect the acoustic power generation. Coltman [3] has conducted measurements of acoustic impedance and pressure. He has shown that the phase relation for acoustic resonance between the jet and acoustic pressure oscillations depends on lip-to-edge distance and the convection velocity of the jet. Yoshikawa et al. [6] has conducted particle image velocimetry and shown that the in-phase relation between the fluctuating jet volume flow into the resonator and the acoustic pressure fluctuations in the resonator is favorable to the acoustic power generation This relation is consistent with the volume-flow model (Helmholtz-Cremer model) [6, 20-25].

Jet angle is an additional parameter in the case of the flute, while the flow channels of the jet in the recorder and organ pipe are usually parallel to the horizontal line of the window. Sawada and Sakaba [26] have investigated the effects of jet angle on the transitions in the sounding mode with an artificial blowing device. Ernoult et al. [27] have studied the effects of the flutist's face inclination on the fundamental frequency and have proposed a formula for the relation between the face inclination angle and the radiation impedance of the flute's window opening. However, the effects of jet angle on harmonic structure have not been quantified and the mechanisms for these effects remain unclear.
The present research focuses on the effects of jet angle on harmonic structure within a practical range estimated on the basis of literature $[11,13,14]$ and experiments in this study. When a human player changes jet angle, other parameters, such as the area that lower lip covers the window of flute, may also change. However, if plural parameters are changed at once, it may be further difficult to know which parameters contribute to the sound and how much. As a first step, experiments are performed under the condition that only a single parameter is changed at once. The effects of jet angle are discussed in conjunction with those of other blowing parameters, such as jet offset. Further, the mechanism for the effects is discussed in terms of both the radiated sound and the flow field measured with a hot-wire anemometer.

The definitions and values of the blowing parameters are shown in Section 2. The actual blowing conditions are measured for a human player by the Schlieren method; the photographic measurement results are presented in Section 3.1. The parameters are varied independently by using an artificial blowing device set up to simulate conditions for a human player, and the radiated sound and flow fields are measured by the methodologies outlined in Section 3.2. The effects of jet angle on harmonic structure are discussed in Section 4.1 along with those of other parameters. The mechanism for the effect of jet angle is discussed in Section 4.2.

\section{Blowing parameters}

The geometrical relation between the jet and the flute depends on jet angle, $\theta_{\mathrm{j}}$, jet offset from the edge, $y_{\mathrm{j}, \mathrm{e}}$, and lip-to-edge distance, $l[11]$. Figure 2 presents the geometrical definitions of these parameters. The jet angle $\left(\theta_{\mathrm{j}}\right)$ is the angle between the horizontal line of the window (mouth opening) and the reference jet direction. This reference jet direction was measured without a flute head joint in the preliminary experiments described below. The jet offset $\left(y_{\mathrm{j}, \mathrm{e}}\right)$ is the distance from the edge to the reference jet direction, where $y_{\mathrm{j}, \mathrm{e}}>0$ means that the reference jet direction is outside the edge. The lip-to-edge distance is the distance from the cavity exit center $\left(o_{\mathrm{j}}\right)$ to the edge. The reference jet direction is the $x_{\mathrm{j}}$-axis, its vertical direction is the $y_{\mathrm{j}}$-axis, and the longitudinal direction of the resonator is the $z_{\mathrm{j}}$-axis. The origin $\left(o_{\mathrm{j}}\right)$ is the center of the upper and lower lips. The values on the axes are nondimensionalized by the throat height of the cavity exit, $h$. The spanwise centers of the window and the cavity exit center $\left(o_{\mathrm{j}}\right)$ are on the same plane $\left(z_{j}=0\right)$, and the above-mentioned parameters are defined on this plane. The acoustic radiation from an isolated flute head joint was investigated. The fundamental frequency of the head joint is around $880 \mathrm{~Hz}$, and this head joint model is the same as in the literature [28].

The reference jet direction is defined as that measured without the head joint through preliminary experiments using the pitot-tube (see Fig. 3). The artificial oral cavity was initially set at the state that the reference line of cavity exit, which connects the tips of the upper and lower lips, 


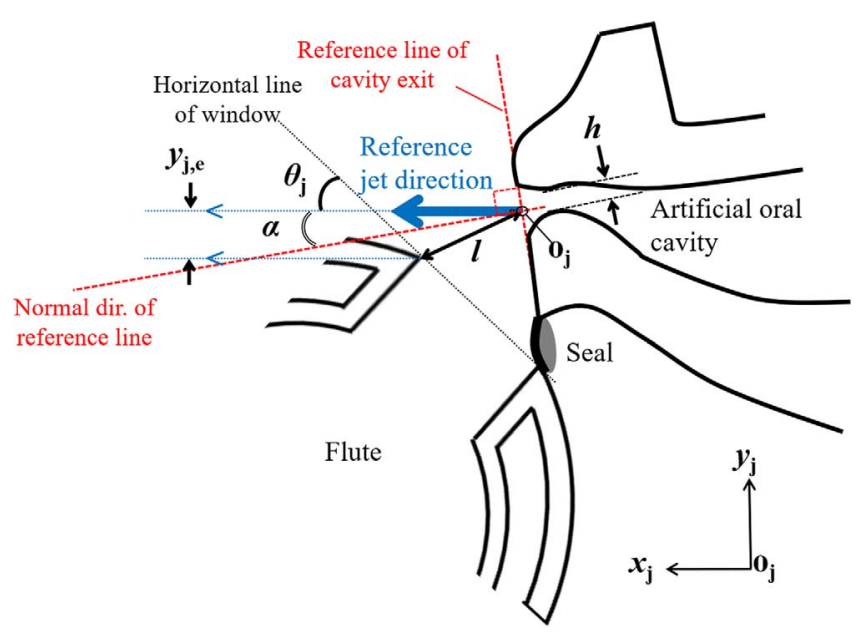

Figure 2. Schematic of blowing parameters with reference jet direction.

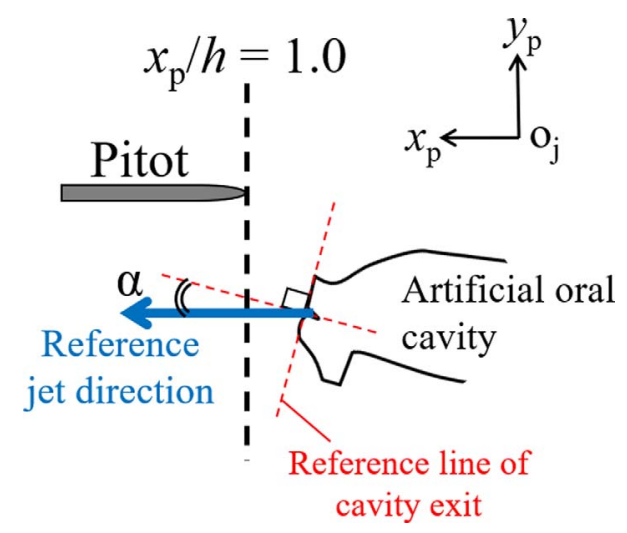

Figure 3. Measurement for reference jet direction.

is parallel to the measurement line $\left(x_{\mathrm{p}} / h=1.0\right)$. The jet velocities were measured by tilting the cavity at an angle, $\alpha$, of $12^{\circ}-15^{\circ}$, which corresponds to changing the angle of the measurement line with respect to the reference line of cavity exit. Figure 4 shows the measured velocity profile. The profile for $\alpha=14^{\circ}$ is symmetrical with respect to $y_{\mathrm{p}} / h=0$, which indicates that the reference jet direction is inclined at $\alpha=14^{\circ}$ to the normal direction of the reference line of cavity exit.

\section{Experimental methods}

\subsection{Measurement of actual blowing condition}

The actual blowing conditions were measured for a human player. Since the blowing conditions can vary depending on several factors, one of the conditions that can occur was investigated.

The jet was visualized by the Schlieren method to estimate the jet angle. Figure 5 illustrates the experimental setup. The Schlieren method allows visualization of the

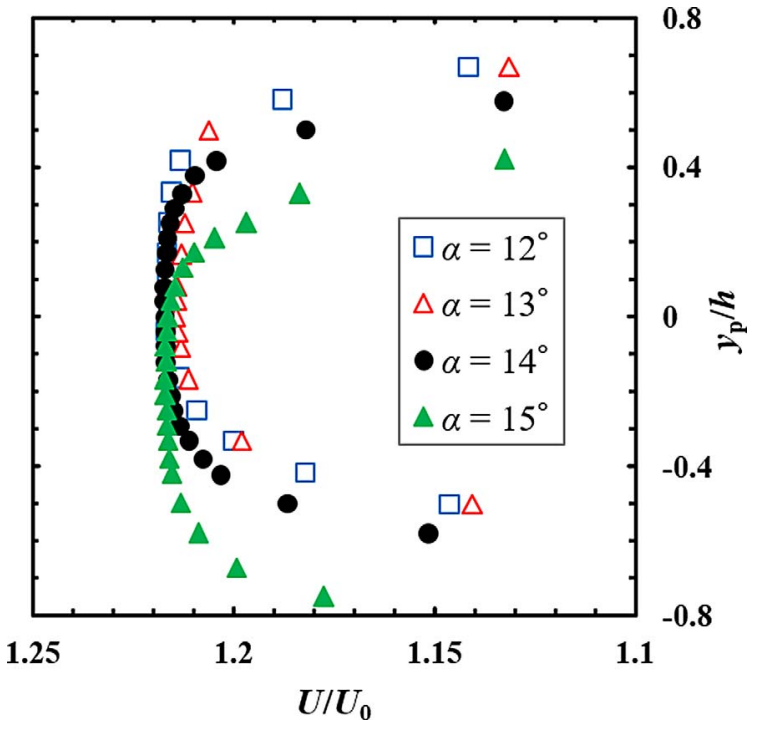

Figure 4. Mean velocity profile of jet measured without flute head joint at $x_{\mathrm{p}} / h=1.0$.

density variation of the flow field, in which the light and dark areas in an image are produced by the variation in the refraction factor of light due to the density variation [29]. A human player blew a flute head joint $4000 \mathrm{~mm}$ from a concave mirror. The beam was reflected on the concave mirror, bent $90^{\circ}$ by a beam splitter, and cut off by a pinhole at the focal point to obtain a sharp and even image. The contrast between light and dark was photographed by a camera.

Figure 6 shows the photograph of a human player blowing at almost $m f$. The head joint was fixed to a jig to ensure that the reference line of window remained parallel to the horizontal line of the window. To estimate the reference jet direction without the head joint, the head joint was slid into the horizontal direction of the window and released from the lips with the player keeping the same blowing state. Schlieren images were taken after the radiated sound was stopped. The jet appears as a black line because of the density gradient produced by the difference in temperature between the air blown by the human and the ambient air. The average jet angle for 6 images was $\theta_{\mathrm{j}}=39^{\circ}$, with a variation, $\theta_{\mathrm{j}, \text { error }}$, of $\pm 2^{\circ}$.

The cavity exit height and lip-to-edge distance were measured from photographs, as shown in Figure 7. In the measurement for the cavity exit height, photographs were taken from the front of the human player blowing the head joint (see Fig. 7a). The height was found to be $h=1.2 \pm 0.1 \mathrm{~mm}$. In the measurement for the lip-to-edge distance, photographs of the human player blowing the fixed head joint were taken from the side (see Fig. $7 \mathrm{~b}$ ). Since the edge center is hidden by the lip plate, the edge center was determined on the basis of the tip position of a 15-mm-heigh strip of masking tape placed at $z_{j}=0$. The lip-to-edge distance was found to be $l / h=5.6 \pm 0.42$. The jet offset, which was calculated geometrically from the jet angle and lip-to-edge distance, was $y_{\mathrm{j}, \mathrm{e}} / h=0.24 \pm 0.25$. 


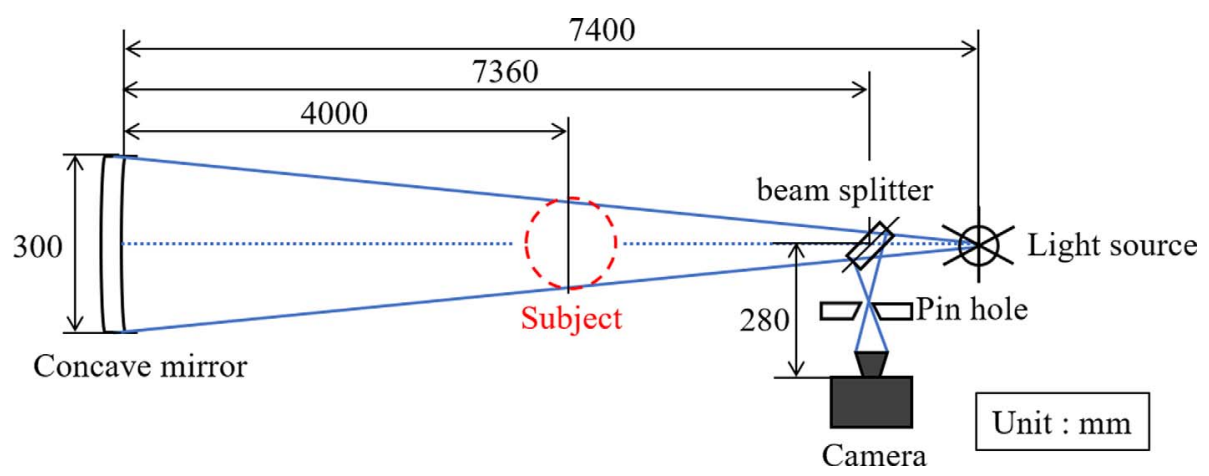

Figure 5. Experimental setup for Schlieren method.

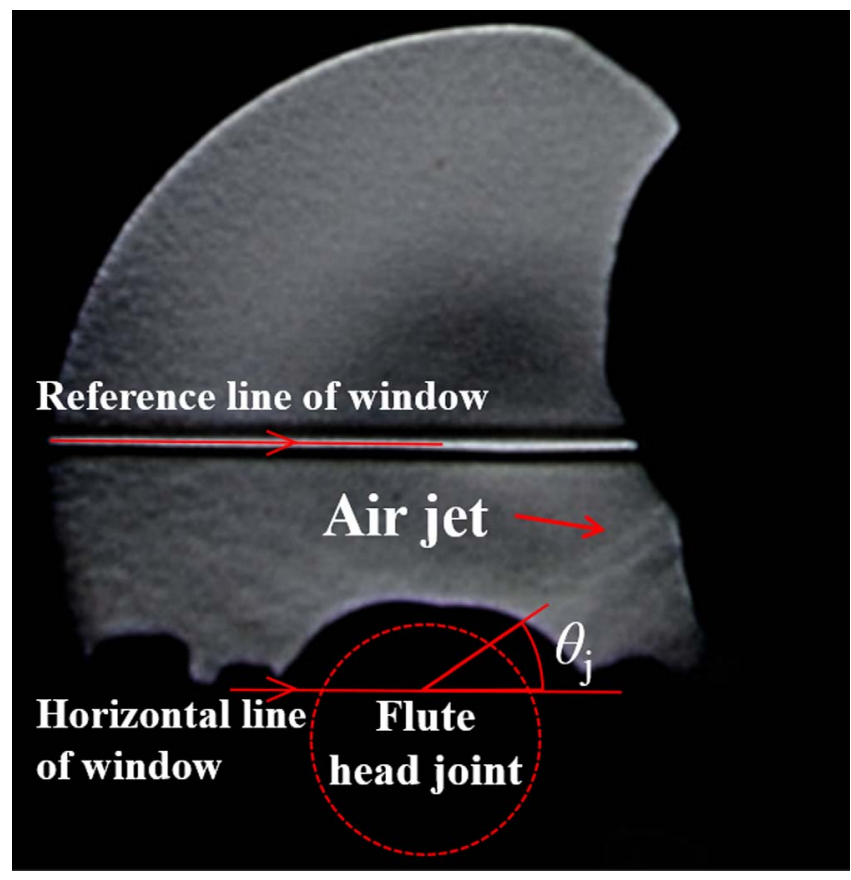

Figure 6. Jet visualization by Schlieren method.

The flow rate $(Q)$ was adjusted so that it gives almost the same fundamental frequency as the human and that the difference of SPL of the second to the third harmonic was the closest to the human within the experimental range.

The actual blowing conditions are shown in Table 1. The radiated sound spectra of the human and device are shown in Figure 8, where the frequency resolution is $1.22 \mathrm{~Hz}$, and the blowing parameters for the device were set to the values in Table 1 . The fundamental frequency was well reproduced by the device. The SPL difference between the second and the third harmonic was larger in the human than that in the device. Within the range of flow rate that the device reproduced the fundamental frequency (16.0-20.0 L/min), the SPL difference was the largest at $Q=16 \mathrm{~L} / \mathrm{min}$; the device's flow rate was set at $Q=16 \mathrm{~L} / \mathrm{min}$. Experiments were performed with reference to the conditions in Table 1 . The values and variations are within the ranges shown in the literature $[11,13,14]$.

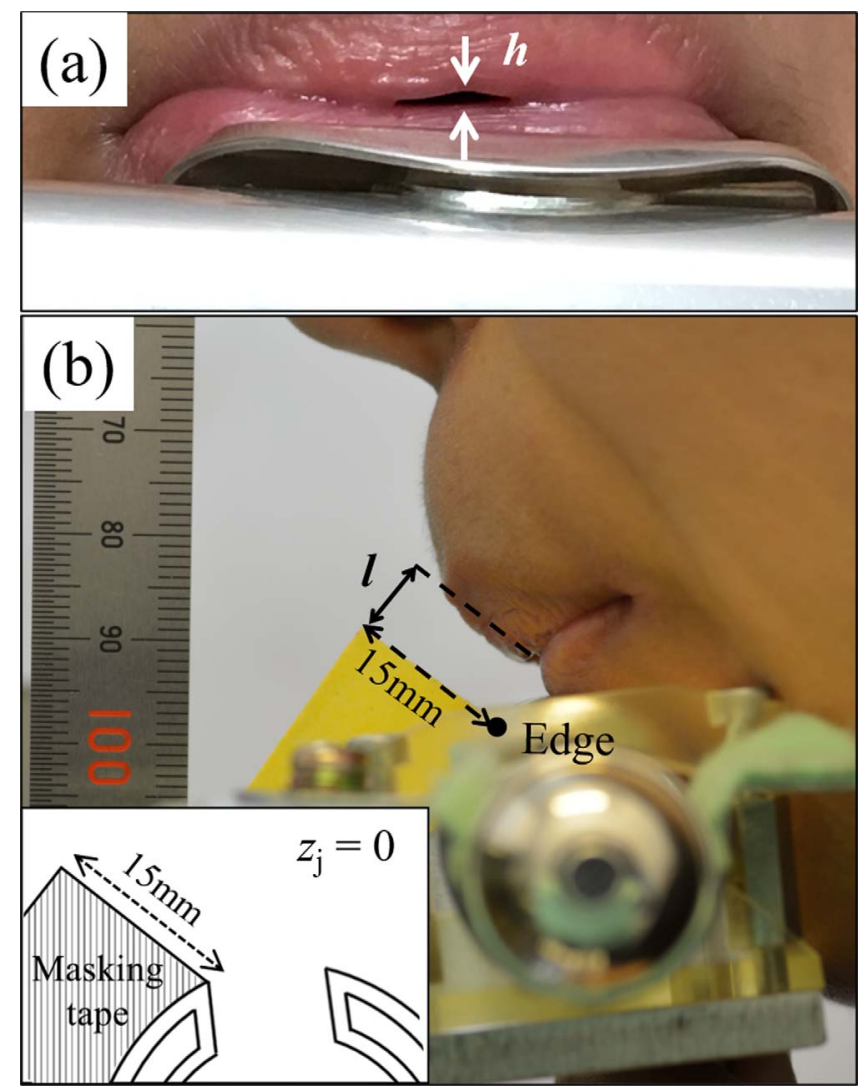

Figure 7. Photographic measurement for cavity exit height (a) and lip-to-edge distance (b).

\subsection{Measurement of radiated sound and flow field using artificial blowing device}

The artificial blowing device [28] shown in Figure 9 was used to vary the blowing parameters independently within the experimental range shown in Table 1. A flute head joint and an artificial oral cavity are installed on the device. The cavity was fabricated with a $3 \mathrm{D}$ printer on the basis of MRI images around the oral cavity of an actual human player blowing a plastic head joint. Since the cavity is made of plastic, shapes of the cavity, including $h$ and $S_{0}$, are constant. In actual performances by a human, the cavity shapes change during playing; however, in this study, the 
Table 1. Actual blowing conditions and experimental ranges.

\begin{tabular}{|c|c|c|c|}
\hline \multirow[t]{2}{*}{ Parameter } & \multicolumn{2}{|c|}{$\begin{array}{l}\text { Actual blowing conditions } \\
\text { of human }\end{array}$} & \multirow[t]{2}{*}{$\begin{array}{l}\text { Experimental } \\
\text { ranges for device }\end{array}$} \\
\hline & Value & Variation & \\
\hline Lip-to-edge distance $l / h$ & 5.6 & \pm 0.42 & $5.0-9.17$ \\
\hline Jet offset $y_{\mathrm{j}, \mathrm{e}} / h$ & 0.24 & \pm 0.25 & $-0.83-1.5$ \\
\hline Flow rate $Q(\mathrm{~L} / \mathrm{min})$ & 16.0 & \pm 2 & $2.0-38.0$ \\
\hline
\end{tabular}

$S_{0}\left(\mathrm{~m}^{2}\right)$ : Cavity exit area.

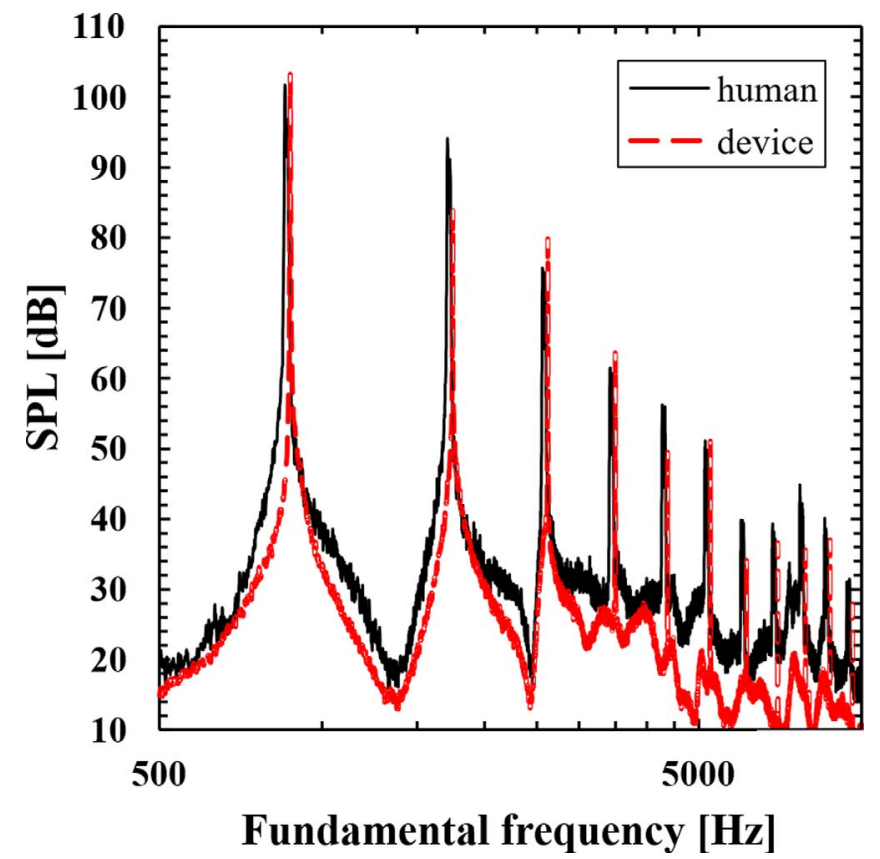

Figure 8. Sound pressure spectra of radiated sound produced by human player and artificial blowing device, where blowing parameters for the device were set to the values in Table 1.

cavity shapes were fabricated to be constant to investigate the effects of parameters independent from the shapes. Between the cavity and the head joint, there is a gap, where the size of gap depends on blowing conditions (see Fig. 9b). In preliminary experiments, the radiated fundamental frequency obtained without covering a seal was about $25 \mathrm{~Hz}$ higher than the fundamental frequency range measured for a human $(875-885 \mathrm{~Hz})$, whereas it was within this range when a seal was placed into the gap. Further, the seal made of aluminum tape and clay was found to be better to reproduce the fundamental frequency range and assure the reproducibility of experiments than the seal made of silicon, clay, and tape. For this reason, the seal made of aluminum tape and clay was placed into the gap. In an actual performance by a human, part of the lower lip interferes with (occludes) the window of the flute. Thus, the opening area of the window depends on blowing conditions. To prevent the change of the opening area affect the

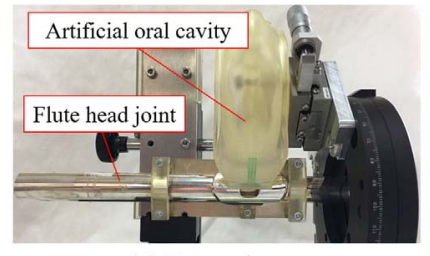

(a) Front view

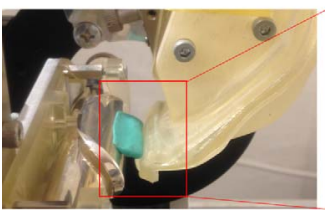

(b) Side view

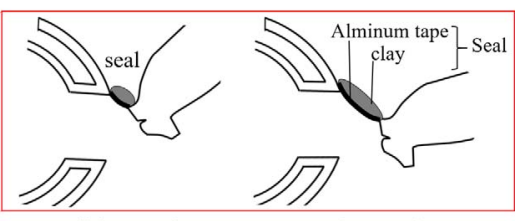

Small jet angle
Large jet angle
Figure 9. Experimental setup for artificial blowing.

radiation impedance around the window [27] and the fundamental frequency, the seal corresponding to the size of gap was positioned so that the opening area was not changed. For three experiments, the reproducibility of sound was about $\pm 4 \mathrm{~Hz}(0.5 \%)$ in terms of the fundamental frequency and about $\pm 1 \mathrm{~dB}$ in terms of the SPLs of the fundamental tone and the second and third harmonics.

Figure 10 illustrates the experimental setup. The air supplied by the compressor (PAOCK SOL-2039) was depressurized to the experimental pressure value by a precision regulator (SMC IR2000). The air was supplied into the artificial oral cavity through a needle valve (KOFLOC 2412), a flowmeter (SMC PFM750S-01-C-M), and a pressure gauge (SMC ZSE30AF-01-C-L). This pressure gauge measures static pressure from the direction that is at right angles to the flow direction.

The radiated sound was measured with a $1 / 2$-inch omnidirectional microphone (RION UC-53A) at $r=100 \mathrm{~mm}$ distance from the resonator end and a precision sound level meter (RION NL-31). The velocity around the window was measured with a hot-wire anemometer. The probe was an I-type probe with a tungsten wire $5 \mu \mathrm{m}$ in diameter and $1 \mathrm{~mm}$ in length. The wire was parallel to the spanwise $\left(z_{\mathrm{j}}\right)$ direction of the cavity exit. Thus, the measured velocity was the absolute value of the synthetic vector of the streamwise $\left(x_{\mathrm{j}}\right)$ and vertical $\left(y_{\mathrm{j}}\right)$ direction velocities. The measurement lines were $x_{\mathrm{j}} / h=1.0,2.0$, and 5.0. The measurement 


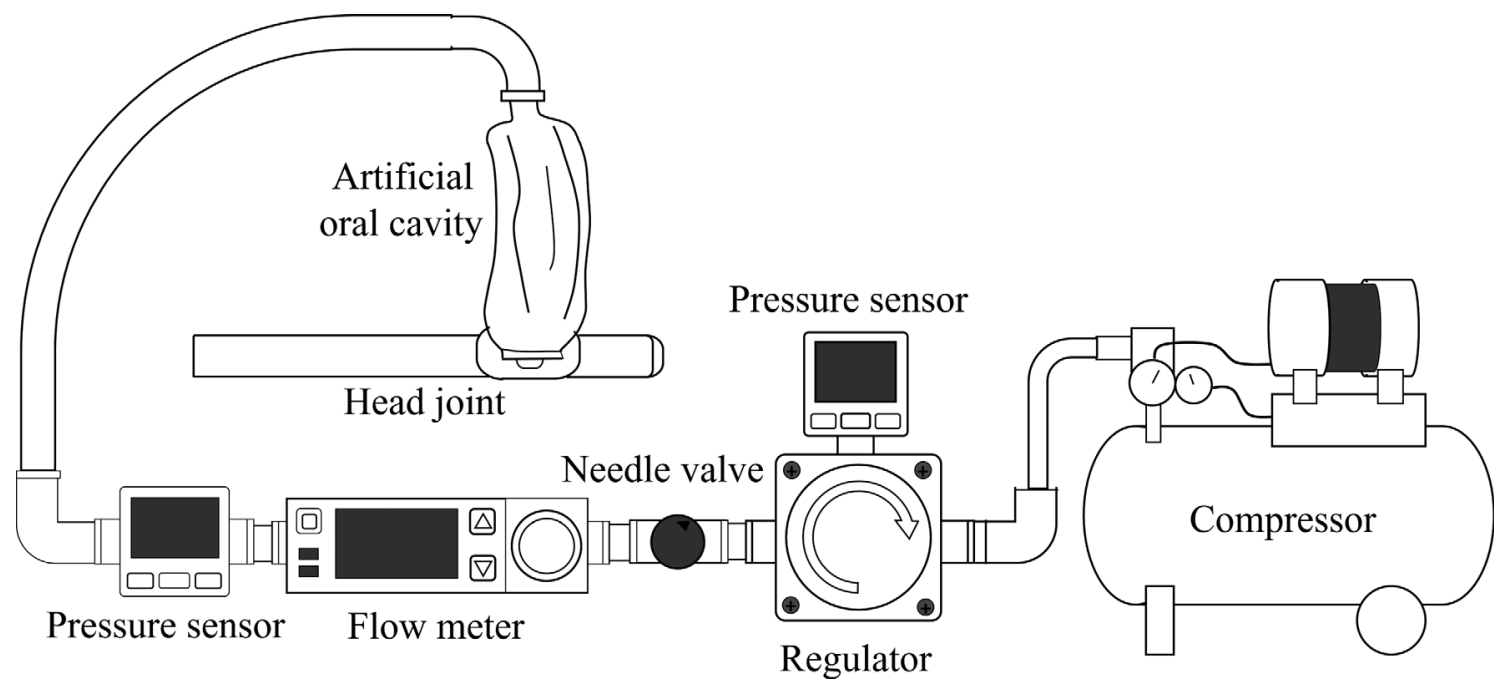

Figure 10. Schematic of artificial blowing.

Table 2. Measurement conditions for radiated sound.

\begin{tabular}{lc}
\hline Condition & Value \\
\hline Sampling frequency $(\mathrm{Hz})$ & 40000 \\
Sampling time $(\mathrm{s})$ & 30 \\
Averaging number $N_{\text {ave }}$ & 72 \\
Frequency resolution for Fourier transform $(\mathrm{Hz})$ & 1.22 \\
\hline
\end{tabular}

conditions for the radiated sound and velocity are shown in Tables 2 and 3. The results of Fourier transform were averaged $N_{\text {ave }}$ times.

\section{Results and discussion}

\subsection{Effects of jet angle and other blowing parameters on sound}

In this section, the effects of blowing parameters, viz., jet offset, lip-to-edge distance, flow rate, and jet angle, on harmonic structure were evaluated by the differential SPL of the second to third harmonic, $\triangle \mathrm{SPL} \equiv \mathrm{SPL}_{2}-\mathrm{SPL}_{3}$. This evaluation is based on the literature [12], which evaluates the difference of SPLs between even and odd harmonics, within the range that the reproducibility of radiated sound was confirmed.

Figure 11 plots the variations in the SPLs of the fundamental tone and harmonics with the blowing parameters, where the arrows indicate practical ranges, the lines represent the actual blowing condition in Table 1, and the second vertical axes show $\Delta \mathrm{SPL}$. All parameters other than the one being varied were fixed to the actual blowing condition. This study defined the practical ranges as that satisfying the following conditions in our experiments within the possible ranges suggested in the literature $[11,13,14]$ : the SPL of the fundamental tone was greater than that of the second harmonic, and the fundamental frequency was
Table 3. Measurement conditions for velocity.

\begin{tabular}{lc}
\hline Condition & Value \\
\hline Minimum resolution of sampling $(\mathrm{mm})$ & $0.07\left(y_{\mathrm{j}} / h=0.058\right)$ \\
Sampling frequency $(\mathrm{Hz})$ & 80000 \\
Sampling time $(\mathrm{s})$ & 30 \\
Averaging number $N_{\text {ave }}$ & 72 \\
Frequency resolution for Fourier & 1.22 \\
transform $(\mathrm{Hz})$ & \\
\hline
\end{tabular}

within \pm 10 cent of that radiated at the actual blowing condition. Compared to the literature [11, 13, 14], the practical ranges are limited, e.g., human players can blow the flute with a lower jet velocity than this range. This is because the practical range was defined for the current experiments under the condition that only a single parameter was varied independently, while the literature $[11,13,14]$ show the ranges for actual human playing.

Figure 11a shows the variation with jet offset. Regarding this variation, the literature [15] has calculated the variation of SPLs of harmonics radiated from a simplified organ pipe model without taking into account of jet deflections. The calculation showed that $\mathrm{SPL}_{2}$ and $\mathrm{SPL}_{3}$ respectively exhibit their minimum and maximum at $y_{\mathrm{j}, \mathrm{e}}=0$ and that as $\left|y_{\mathrm{j}, \mathrm{e}}\right|$ increases (the jet fluctuates more asymmetrically to edge), $\mathrm{SPL}_{2}$ and $\mathrm{SPL}_{3}$ respectively increase and decrease. In Figure 11a, the second harmonic curve has a minimum around $y_{\mathrm{j}, \mathrm{e}}=0$, which is consistent with the calculation. Unlike the calculation, the third harmonic curve here is not symmetrical with respect to $y_{\mathrm{j}, \mathrm{e}}=0$. In $y_{\mathrm{j}, \mathrm{e}}>0$ (the reference jet direction is directed to outside the edge), $\mathrm{SPL}_{3}$ decreases as $\left|y_{\mathrm{j}, \mathrm{e}}\right|$ increases, while $\mathrm{SPL}_{3}$ remains almost constant in $y_{\mathrm{j}, \mathrm{e}}<0$. Although the SPLs depend only on $\left|y_{\mathrm{j}, \mathrm{e}}\right|$ in the calculation [15], in the actual blowing, $\mathrm{SPL}_{3}$ seems to decrease less when the jet fluctuates inside the edge $\left(y_{\mathrm{j}, \mathrm{e}}<0\right)$ than when it fluctuates outside. This may be because of incomplete symmetry in the actual 


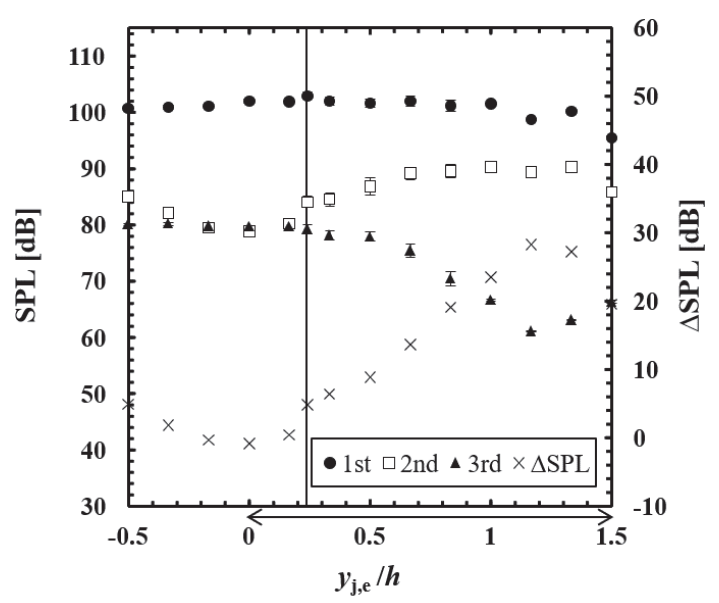

(a) Variation with jet offset

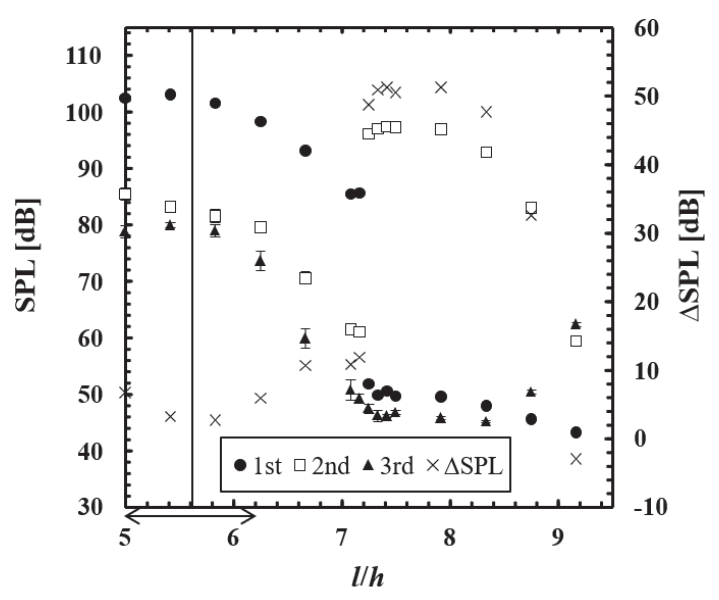

(b) Variation with distance

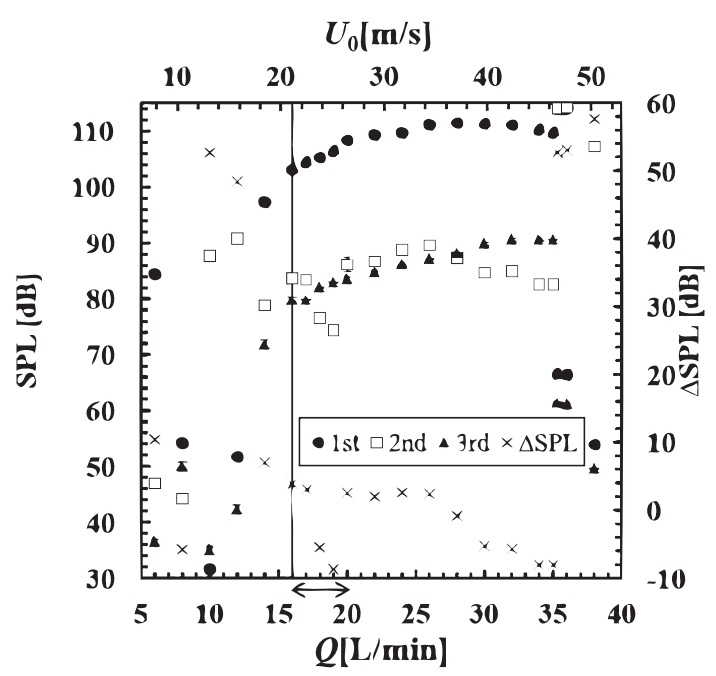

(c) Variation with flow rate

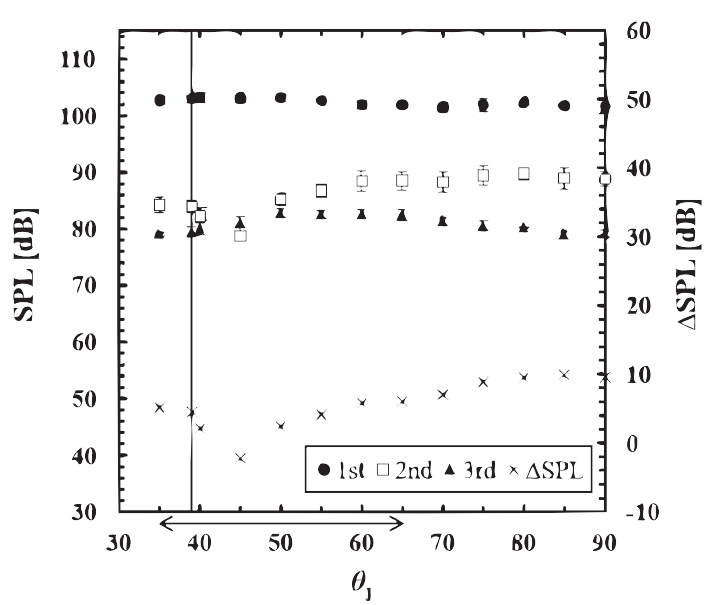

(d) Variation with jet angle

Figure 11. Variations of harmonic structure with blowing parameters. Lines and arrows show actual blowing conditions and practical ranges, respectively. All parameters other than the one being varied were fixed to the actual blowing conditions shown in Table $1\left(\theta_{\mathrm{j}}=39^{\circ}, y_{\mathrm{j}, \mathrm{e}}=0.24, l / h=5.6, Q=16 \mathrm{~L} / \mathrm{min}\right)$. Error bar is shown for each measured value. In (a), $y_{\mathrm{j}, \mathrm{e}}>0$ means that the reference jet direction is directed to outside the edge.

velocity profile of the jet around the edge, as to be shown in Figure 14b of Section 4.2, while the calculation assumes a velocity profile symmetrical with respect to the jet center. In the practical range, $\Delta$ SPL significantly increases from about $-1 \mathrm{~dB}$ to $28 \mathrm{~dB}$ with increasing jet offset in $0 \leq y_{\mathrm{j}, \mathrm{e}} / h \leq 1.2$.

Figure 11b shows that the SPLs of the fundamental tone and the harmonics decrease with increasing lip-to-edge distance in $5.8 \leq l / h \leq 7.1$ and that the fundamental frequency then shifts from the first to the second mode at around $l / h=7.2$, which is outside the practical range. The phase of vertical fluctuations of the jet delays as the jet travels downstream. This time delay (convection time) from the cavity exit to the edge can be approximated by $l / U_{\mathrm{c}}$, where $U_{\mathrm{c}}$ is the convection velocity. When $l$ exceeds a certain length, the convection time of jet deviates the condition that the jet fluctuation at the edge can keep the favorable phase relation with the pressure fluctuations in the first mode, then the fundamental shifts to the second mode.

Figure 11c shows that the SPLs of the fundamental tone and the third harmonic increase with increasing flow rate in the practical range, while $\mathrm{SPL}_{2}$ decreases for $16 \leq Q \leq$ $19 \mathrm{~L} / \mathrm{min}$ and increases for $19 \leq U_{0} \leq 20 \mathrm{~L} / \mathrm{min}$. With the exception of this local variation around $Q=19 \mathrm{~L} / \mathrm{min}$, the second harmonic also increases around the practical range. As flow rate increases, the kinetic energy supplied from the jet to the acoustic field in the resonator increases, and the convection velocity, $U_{c}$, also increases. For flow rate, both the supplied kinetic energy and the phase relation can affect the SPL. However, as for the local decrease of the second harmonic around $Q=19 \mathrm{~L} / \mathrm{min}$, it cannot be explained by the kinetic energy, because the energy increases continuously with $Q$. To investigate the phase relation, the 
Table 4. Variation of $\Delta \mathrm{SPL}$ with parameters in practical range.

\begin{tabular}{lccc}
\hline Parameter & \multicolumn{3}{c}{$\begin{array}{c}\text { Range of } \Delta \text { SPL in } \\
\text { practical range }(\mathrm{dB})\end{array}$} \\
\cline { 2 - 4 } & Min. & Max. & Diff. \\
\hline Jet angle $\theta_{\mathrm{j}}$ & -2.2 & 6.1 & 8.3 \\
Lip-to-edge distance $l / h$ & 2.6 & 6.7 & 4.0 \\
Jet offset $y_{\mathrm{j}, \mathrm{e}} / h$ & -0.8 & 28.3 & 29.1 \\
Flow rate $Q$ & -5.5 & 3.9 & 9.4 \\
\hline
\end{tabular}

nondimensional convection time of the second mode of jet oscillation, $\left(l / U_{\mathrm{c}}\right) / T_{2}=t_{\mathrm{c}, 2} / T_{2}$, was estimated: $t_{\mathrm{c}, 2} / T_{2}$ for $Q=18,19$ and $20 \mathrm{~L} / \mathrm{min}$ were $0.65,0.61$ and 0.58 , respectively. Here, the convection velocity was assumed to be $U_{\mathrm{c}}=0.77 U_{0}$ based on the estimation in Section 4.2, and $T_{2}$ is the period of the second harmonic frequency measured at each flow rate. The convection time approaches to 0.5 with increasing flow rate in $18-20 \mathrm{~L} / \mathrm{min}$. The convection time is to be almost 0.5 (half a period) to form the favorable phase relation between the jet and the pressure fluctuations [7-9]. The phase relation approaches the favorable condition and seems not to be the cause of the local decrease of $\mathrm{SPL}_{2}$ around $Q=19 \mathrm{~L} / \mathrm{min}$. Another possible factor is to be discussed in Section 4.2. Except for $Q=18$ and $19 \mathrm{~L} / \mathrm{min}$, $\Delta$ SPL remains almost constant in the practical range.

Figure 11d shows that the SPL of the fundamental tone varies little with jet angle; thus, the phase relation for the acoustic power generation seems to be largely unaffected. $\mathrm{SPL}_{2}$ decreases with increasing jet angle for $\theta_{\mathrm{j}}<45^{\circ}$, while it increases for $\theta_{\mathrm{j}}>45^{\circ}$. SPL $\mathrm{SParies}_{2}$ about $10 \mathrm{~dB}$ with jet angle, while $\mathrm{SPL}_{3}$ varies about $3 \mathrm{~dB}$. Since $\Delta \mathrm{SPL}$ varies mainly due to the variation of $\mathrm{SPL}_{2}$ under this condition, $\triangle \mathrm{SPL}$ exhibits its minimum at $\theta_{\mathrm{j}}=45^{\circ}$.

Table 4 lists the variation of $\Delta$ SPL with the parameters in the practical ranges. $\triangle$ SPL variations with jet offset and lip-to-edge distance are about 30 and $5 \mathrm{~dB}$, respectively, while the variations with jet angle and flow rate are both around $10 \mathrm{~dB}$. The harmonic structure varied markedly with jet offset, almost equally with jet angle and flow rate, but comparatively less with lip-to-edge distance. The effect of jet angle on timbre seems to be an important consideration for flute players.

In Figure 11, the blowing conditions other than the variable one were fixed to the actual blowing condition measured for a human player. To confirm generality of the effects of jet angle on harmonic structure, Figure 12 shows the variations measured by fixing jet offset to different values from that in Table $1\left(y_{\mathrm{j}, \mathrm{e}} / h=0.24\right): y_{\mathrm{j}, \mathrm{e}} / h=0.0$, $0.17,1.0$. The jet angle that $\Delta$ SPL exhibits its minimum is $\theta_{\mathrm{j}, \text { min }}=37^{\circ}$ for $y_{\mathrm{j}, \mathrm{e}} / h=0.17$ and $\theta_{\mathrm{j}, \text { min }}=45^{\circ}$ for $y_{\mathrm{j}, \mathrm{e}} / h=$ 0.24 . As $y_{\mathrm{j}, \mathrm{e}} / h$ decreases (increases), the curve of $\Delta \mathrm{SPL}$ seems to shift toward a smaller (larger) jet angle, and $\theta_{\mathrm{j}}$, $\min _{\text {min }}$ seems to become small (large). For $y_{\mathrm{j}, \mathrm{e}} / h=1.0, \Delta \mathrm{SPL}$ decreases with increasing jet angle, which seems because the curve shift toward a larger jet angle and $\theta_{\mathrm{j}, \text { min }}$ becomes larger than the jet angle in the experimental range. $\Delta \mathrm{SPL}$ variations in the practical range are about $12 \mathrm{~dB}, 8 \mathrm{~dB}$, and $17 \mathrm{~dB}$ for $y_{\mathrm{j}, \mathrm{e}} / h=0.0,0.17$, and 1.0 , respectively.
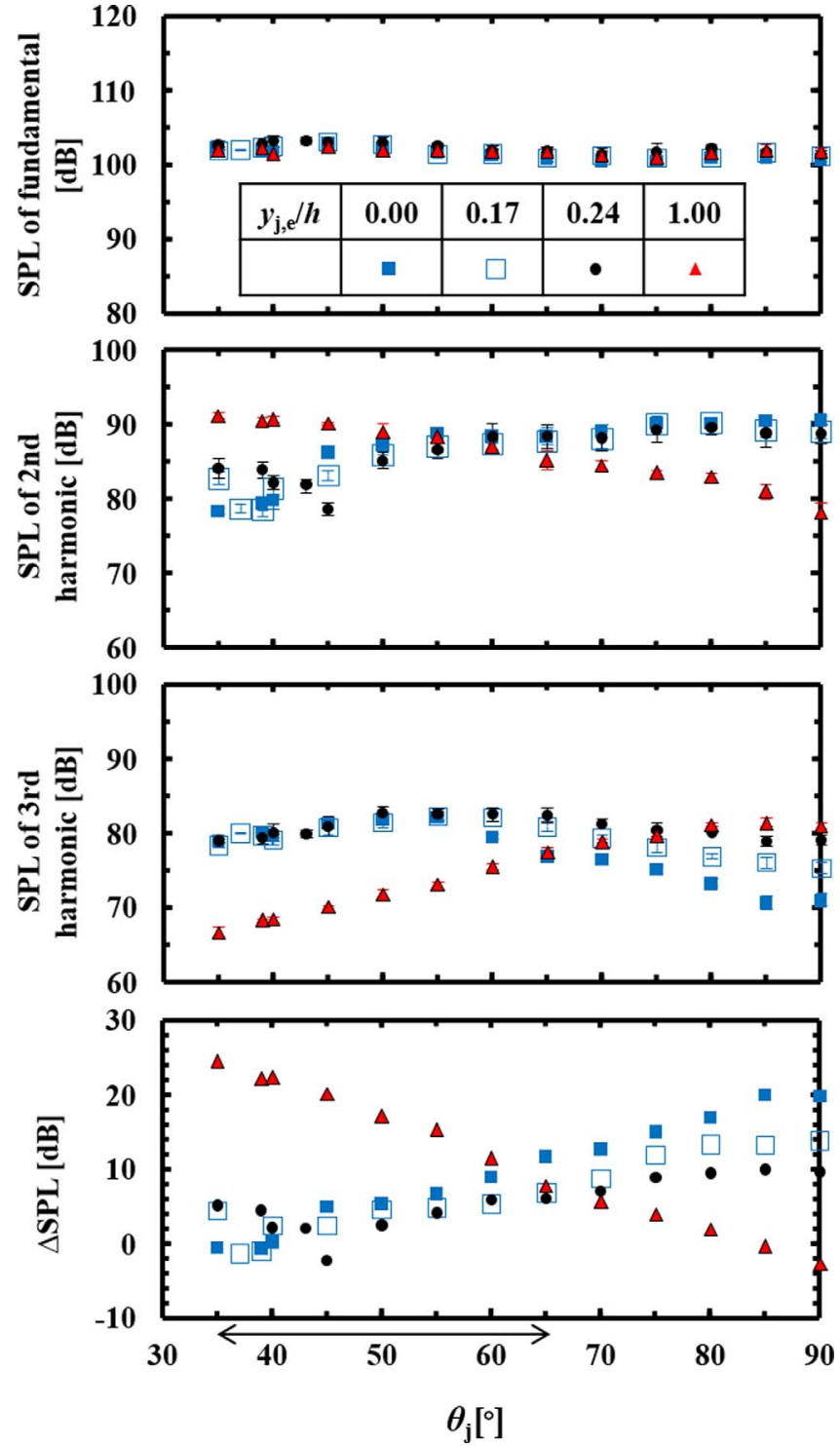

Figure 12. Variations of harmonic structure with jet angle, where lip-to-edge distance and flowrate were fixed to the actual blowing conditions shown in Table $1(l / h=5.6, Q=16 \mathrm{~L} / \mathrm{min})$. Arrow shows practical range.

$\Delta$ SPL varies with jet angle probably in the range of not exceeding the variation with jet offset. (See Appendix for the variations measured for a flute with a body and a foot joint.)

\subsection{Mechanism whereby jet angle affects harmonic structure}

In the previous section, variations in harmonic structure were discussed with the blowing parameters defined on the basis of the reference jet direction measured without the flute head joint. With the head joint, the actual jet direction can be different from those measured without the head joint, which may affect the resulting jet offset. In this section, the actual jet offset is estimated on the basis of both the radiated sound and the flow field for the jet 

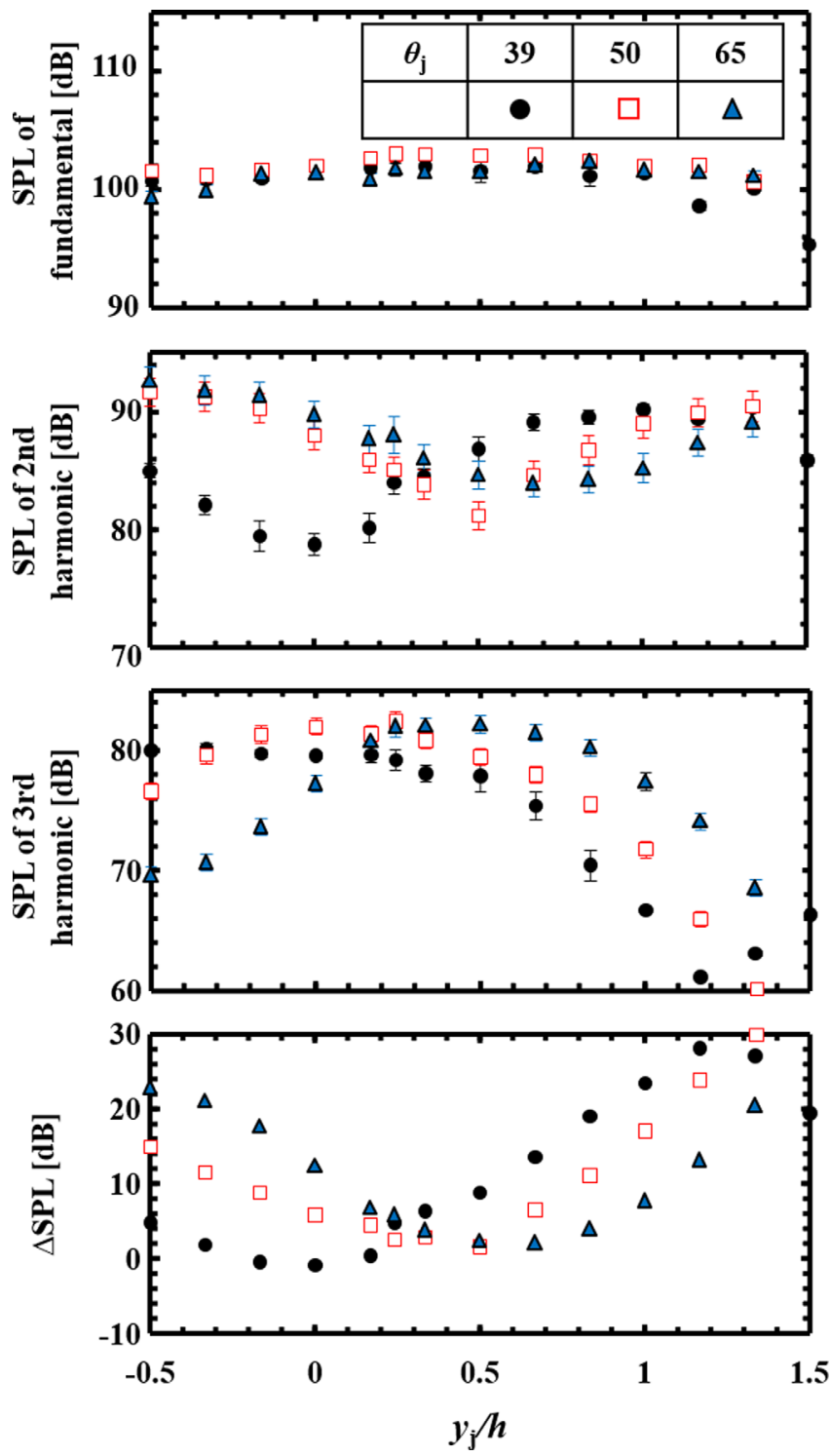

Figure 13. Variations of harmonic structure with jet offset, where lip-to-edge distance and flowrate were fixed to the actual blowing conditions shown in Table $1(l / h=5.6, Q=16 \mathrm{~L} / \mathrm{min})$.

angles $\theta_{\mathrm{j}}=39^{\circ}, 65^{\circ}$, and $50^{\circ}$, which are respectively the jet angles at the actual blowing condition, the maximum jet angle in the practical range, and a jet angle between these two.

Figure 13 plots the variation in the SPLs of the harmonics with jet offset. For $\theta_{\mathrm{j}}=39^{\circ}, 50^{\circ}$, and $65^{\circ}$, the jet offsets minimizing the $\Delta \mathrm{SPL}, y_{\min } / h$, were about $0.0,0.5$, and 0.7 , respectively. As the jet angle increases, $y_{\min } / h$ becomes large. This implies that the second and third harmonic curves shift toward the positive direction.

To elucidate this change in $y_{\mathrm{min}} / h$ from the flow field, the periodical variations of velocity profiles in Figure 14 were obtained by phase-averaging the values measured with a hot-wire anemometer with reference to the sound pressure fluctuations. In these measurements, the jet offset was set to zero $\left(y_{\mathrm{j}, \mathrm{e}} / h=0\right)$, i.e., the reference jet direction without the head joint was directed toward the edge. The radiated SPLs are shown in Figure 12. In a preliminary operation, the phase of the sound pressure fluctuation in the resonator, phase $[p(t)]$, was calculated from the radiated sound pressure fluctuation based on the distance from the measurement point to the open end of the resonator $\left(z_{\mathrm{j}}=L\right) . t=0$ is the instant when the fundamental mode of phase $[p(t)]$, phase $\left[p_{1}(t)\right]$, exhibits a minimum, where the fluctuation with the fundamental mode was extracted from phase $[p(t)]$ by the procedure described in the literature [19]. Figure 14 shows that the velocity profiles at $x_{\mathrm{j}} / h=1$ have top-hat-shapes (shapes similar to rectangle functions), while the computation [19] showed parabolic profiles at the jet exit of the recorder under almost the same $U_{0}$ as this study. Compared to the channel in the recorder, the channel is shorter in the current oral cavity; therefore, the shear layer forms thinner, and the profiles have top-hat-shapes. The profiles are temporally inclined outward $\left(y_{\mathrm{j}}>0\right)$ or inward $\left(y_{\mathrm{j}}<0\right)$ probably affected by the acoustic feedback from the acoustic field, as also observed around the jet exit in the computation [19]. Their fluctuations are amplified at $x_{\mathrm{j}} / h=5$. The line in each profile shows the jet displacement, $y_{\mathrm{d}}$, where $y_{\mathrm{d}}$ was defined in this study as the center of the half-value positions of maximum velocity. $y_{\mathrm{d}}$ fluctuates between the outside and inside during each period.

Figure 15 shows the periodical variations of the jet displacements at $x_{\mathrm{j}} / h=1.0,2.0$, and 5.0, as well as phase $[p(t)]$. The instantaneous jet displacement fluctuates almost sinusoidally at $x_{\mathrm{j}} / h=1$ and 2 . Further downstream, the instant that the jet displacement changes from increasing (decreasing) to decreasing (increasing) is delayed owing to the convection of the jet fluctuations. The convection velocity of the first mode of jet fluctuations, $U_{\mathrm{c}, 1}$, was calculated from the phase difference of the first modes of $y_{\mathrm{d}}(t)$ between $x_{\mathrm{j}} / h=1$ and 5 . The convection velocities were $U_{\mathrm{c}, 1}=16.6 \mathrm{~m} / \mathrm{s}\left(0.77 U_{0}\right)$ for $\theta_{\mathrm{j}}=39^{\circ}$ and $50^{\circ}, U_{\mathrm{c}, 1}=$ $19.8 \mathrm{~m} / \mathrm{s}\left(0.92 U_{0}\right)$ for $\theta_{\mathrm{j}}=65^{\circ}$. The convection velocity nondimensionalized by the maximum velocity at the exit, $U_{\mathrm{c}, 1} / U_{0, \max }$, were respectively estimated to be 0.34 for $\theta_{\mathrm{j}}=39^{\circ}$ and $50^{\circ}, 0.65$ for $\theta_{\mathrm{j}}=65^{\circ}$, which are almost within the values in the literature $[13,19,30,31]$. Here, $U_{0, \max }$ is estimated from the reduction ratio of the maximum jet velocity between $x_{\mathrm{j}} / h=1$ and $2,\left(U_{1, \max }-U_{2, \max }\right) / h$, since $U_{0 \text {,max }}$ is unmeasurable because the cavity exit is too narrow to insert the probe. The measurements in the literature [13] showed that the convection velocity depends mostly on the shear layer formation in the channel and increases when the jet gets sharper. Figure 16 shows the time-averaged velocity profile near the cavity exit $\left(x_{\mathrm{j}} / h=1.0\right)$, where the vertical axes show the relative height to the jet displacement. The boundary layer at $\theta_{\mathrm{j}}=65^{\circ}$ is thinner than at $\theta_{\mathrm{j}}=39^{\circ}$, $50^{\circ}$.; therefore, $U_{\mathrm{c}, 1} / U_{0, \max }$, for $\theta_{\mathrm{j}}=65^{\circ}$ is the largest among the jet angles. The convection velocities are consistent with the velocity profile. This confirms that the periodical jet fluctuations obtained from the measurements with the hot-wire anemometer are a reasonable representation. The velocity profile near the exit changes with the jet angles probably because the jet may be affected by the acoustic pressure fluctuations. With increasing jet angle, the channel 

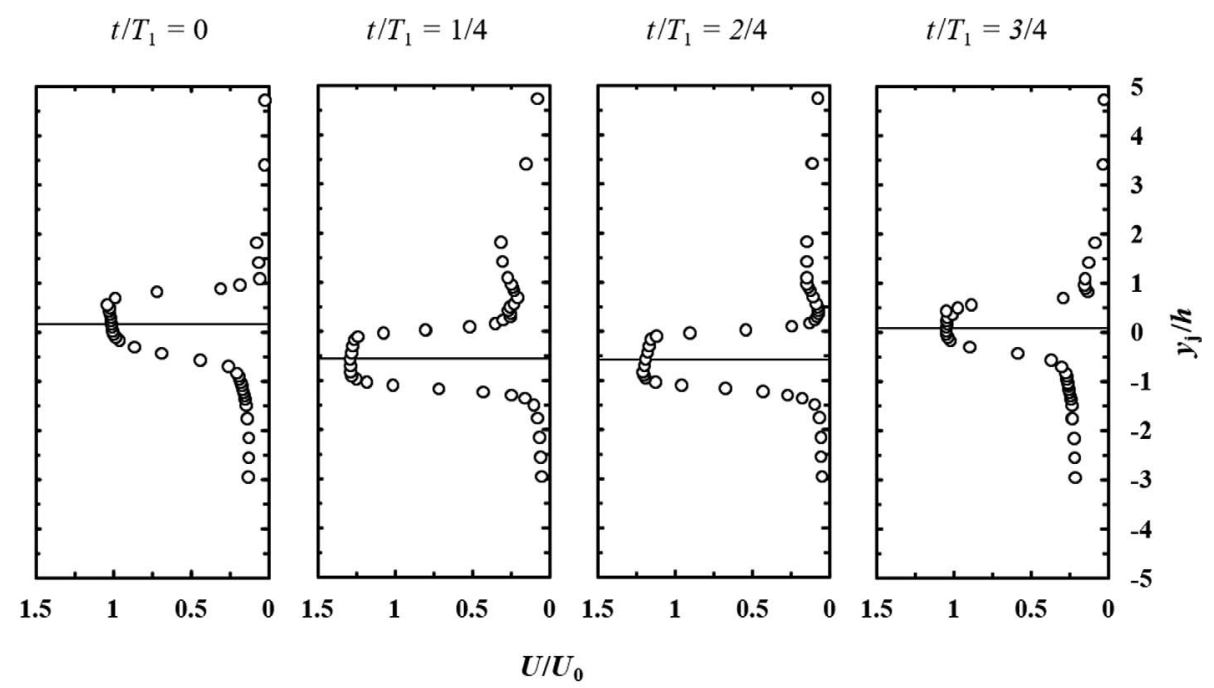

(a) $x_{\mathrm{j}} / h=1.0$
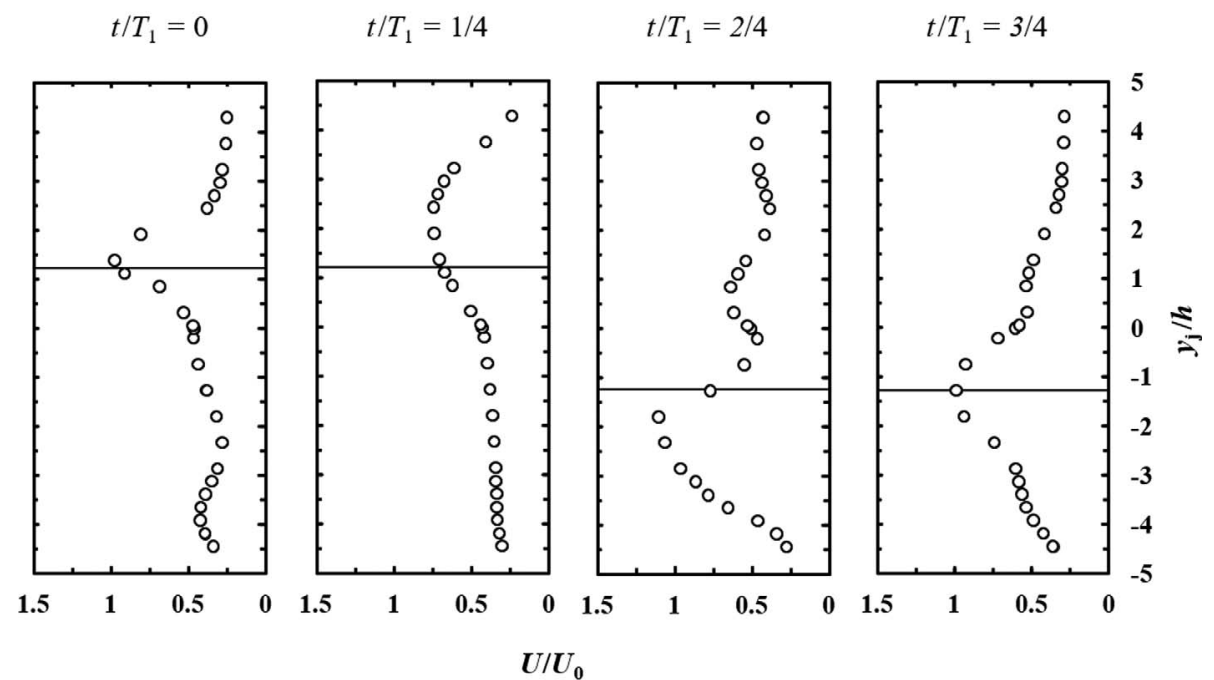

(b) $x_{\mathrm{j}} / h=5.0$

Figure 14. Periodical variations of velocity profiles, where lines show jet displacements $\left(y_{\mathrm{d}}\right) \cdot\left(\theta_{\mathrm{j}}=39^{\circ}, y_{\mathrm{j}, \mathrm{e}}=0, l / h=5.6\right.$, $Q=16 \mathrm{~L} / \mathrm{min})$.

becomes more vertical to the window (see Fig. 9b), which may change the effect of the acoustic pressure fluctuations on the jet.

Assuming that $U_{\mathrm{c}, 1}$ is constant between $x_{\mathrm{j}} / h=1$ and $l / h$, the phase of jet displacement fluctuations with the fundamental mode at the edge $\left(x_{\mathrm{j}} / h=l / h\right)$, phase $\left[y_{\mathrm{d}, 1}(t)\right]$, were calculated for each jet angle (see Fig. 17). The phase differences of the jet displacement to the pressure fluctuations were $115^{\circ}, 130^{\circ}$, and $135^{\circ}$, for $\theta_{\mathrm{j}}=39^{\circ}, 50^{\circ}$, and $65^{\circ}$, respectively. Although $U_{\mathrm{c}, 1}$ is almost the same for $\theta_{\mathrm{j}}=39^{\circ}$ and $50^{\circ}$ and the largest for $\theta_{\mathrm{j}}=65^{\circ}$, the resulting phase difference increases with increasing jet angle and is not determined only from $U_{\mathrm{c}, 1}$. This seems to because the phase difference depends both on $U_{c, 1}$ and the time delay from the jet receives acoustic feedback until it appears in jet fluctuations at the exit [19]. The phase differences, $115^{\circ}-135^{\circ}$, are within the threshold for the acoustic power generation at $90^{\circ}-270^{\circ}$ [20]. Jet angle seems not to effect significantly on the condition for the acoustic power generation.

To estimate the actual jet offset, the jet fluctuation center, $y_{\mathrm{fc}}\left(x_{\mathrm{j}}\right)$, was defined in this study as the time average of jet displacement fluctuation $\left(y_{\mathrm{d}}\right)$. Figure 18 plots the spatial distribution of the jet fluctuation centers, where the horizontal axis is shown from the cavity exit $\left(x_{\mathrm{j}}=0\right)$ to the edge $\left(x_{\mathrm{j}}=l=5.6 \mathrm{~h}\right)$. The jet fluctuation centers at $x_{\mathrm{j}}=0, y_{\mathrm{fc}}(0)$, seem not to coincidence with the geometrical origin $\left(y_{\mathrm{fc}}=0\right)$ and they vary with jet angle. Assuming that the jet travels with the same deflection from $x_{\mathrm{j}} / h=0$ to 2 , $y_{\mathrm{fc}}(0)$ is around -0.25 for $\theta_{\mathrm{j}}=39^{\circ}$ and $50^{\circ}$, and -0.45 for 


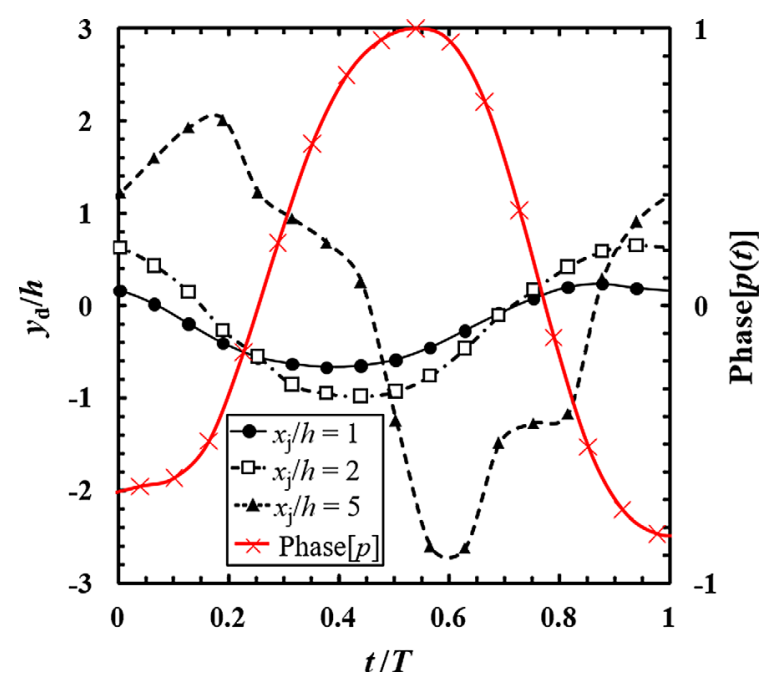

Figure 15. Periodical variations of jet displacements at $x_{\mathrm{j}} / h=1,2$, and 5 , as well as periodical sound pressure fluctuation in the resonator. $\left(\theta_{\mathrm{j}}=39^{\circ}, y_{\mathrm{j}, \mathrm{e}}=0, l / h=5.6, Q=16 \mathrm{~L} / \mathrm{min}\right)$.

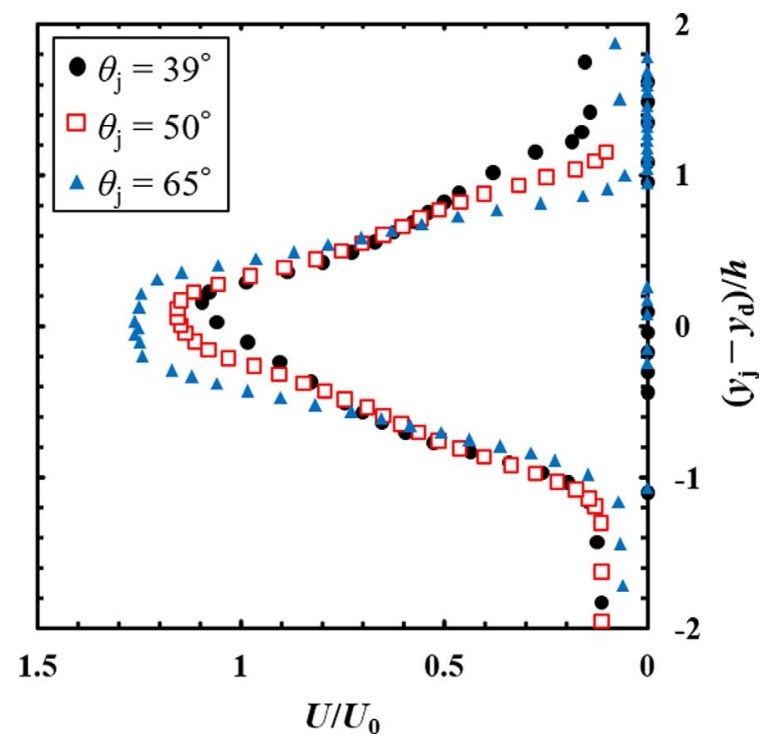

Figure 16. Time averaged velocity profiles at $x_{\mathrm{j}} / h=1$, where vertical axes show relative height to jet displacement. $\left(y_{\mathrm{j}, \mathrm{e}}=0\right.$, $l / h=5.6, Q=16 \mathrm{~L} / \mathrm{min})$.

$\theta_{\mathrm{j}}=65^{\circ} \cdot y_{\mathrm{fc}}(0)$ locates further inside $\left(y_{\mathrm{fc}}<0\right)$ at the jet angle around the upper limit of the practical range.This is presumably because the jet fluctuates in the channel $\left(x_{\mathrm{j}} \leq 0\right)$ affected by the acoustic pressure fluctuations. In the upstream half of the window $\left(0<x_{\mathrm{j}}<0.5 l\right), y_{\mathrm{fc}}(h)$ is almost the same at $\theta_{\mathrm{j}}=39^{\circ}$ and $50^{\circ}$ though, $y_{\mathrm{fc}}(2 h)$ is farther outside at $\theta_{\mathrm{j}}=39^{\circ}$ than at $\theta_{\mathrm{j}}=50^{\circ}$. This indicates that the jet is deflected in the upstream. As the jet travels downstream from $x_{\mathrm{j}}=2 h$ to $x_{\mathrm{j}}=5 h$, it is deflected outside the resonator $\left(y_{\mathrm{fc}}>0\right)$ at $\theta_{\mathrm{j}}=39^{\circ}$, while it is deflected inside at $\theta_{\mathrm{j}}=50^{\circ}$ and $65^{\circ}$. The jet deflections at $\theta_{\mathrm{j}}=50^{\circ}$ and $65^{\circ}$

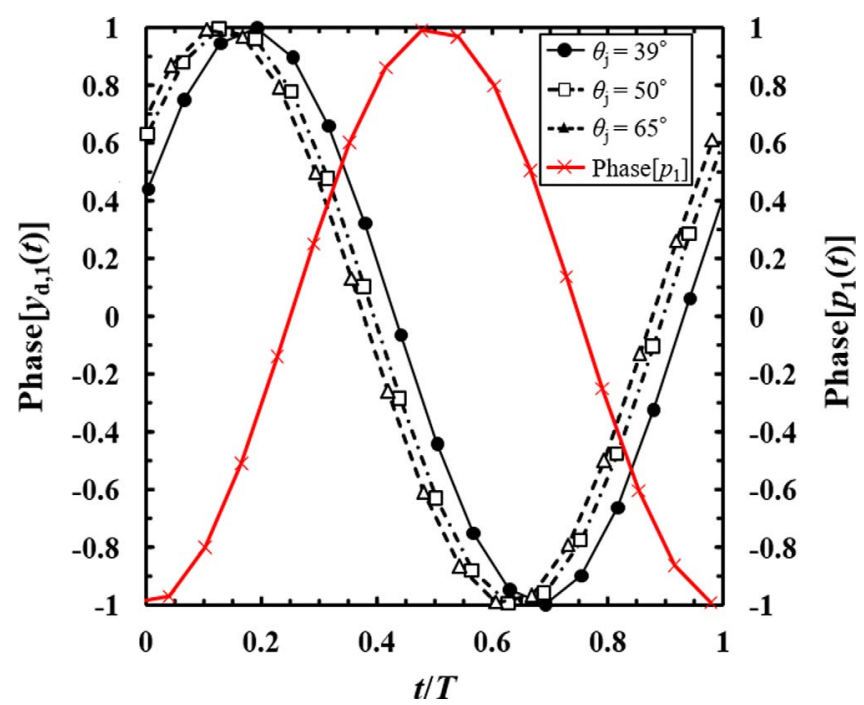

Figure 17. Periodical variations of jet at the edge $\left(x_{\mathrm{j}} / h=l / h\right)$ and pressure fluctuations at the fundamental mode. $\left(y_{\mathrm{j}, \mathrm{e}}=0\right.$, $l / h=5.6, Q=16 \mathrm{~L} / \mathrm{min})$.

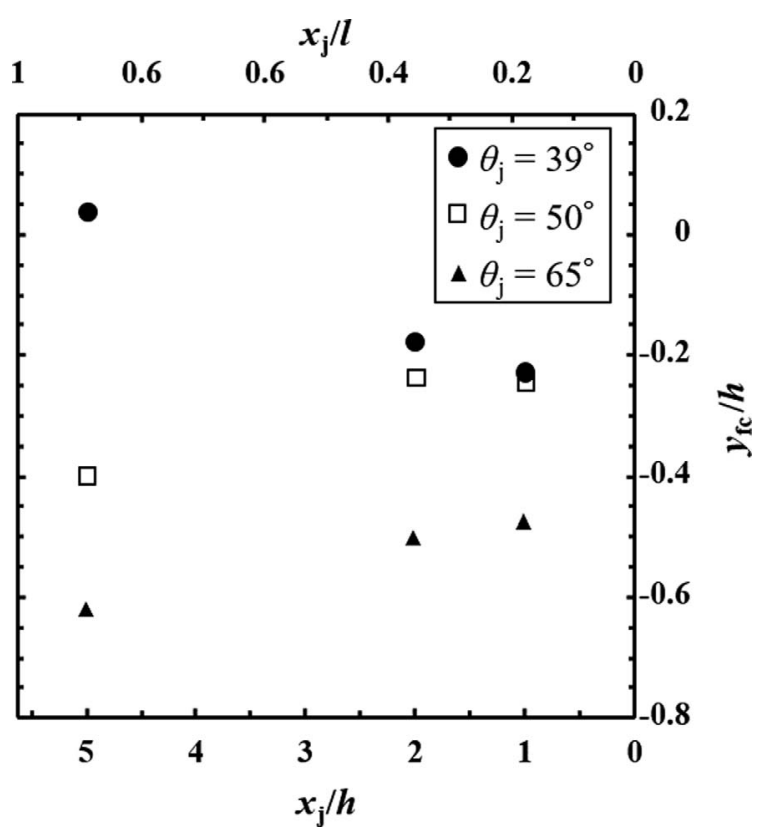

Figure 18. Spatial distributions of jet fluctuation centers. $\left(y_{\mathrm{j}, \mathrm{e}}=0, l / h=5.6, Q=16 \mathrm{~L} / \mathrm{min}\right)$.

are almost the same. The difference of $y_{\mathrm{fc}}(5 h)$ between $\theta_{\mathrm{j}}=50^{\circ}$ and $65^{\circ}$ seems to be due to the difference of jet fluctuation centers at the cavity exit $\left(x_{\mathrm{j}}=0\right)$, while the difference between $\theta_{\mathrm{j}}=39^{\circ}$ and $50^{\circ}$ is due to the jet deflection in the window $\left(0<x_{\mathrm{j}}\right)$. The jet fluctuation centers near the edge probably depend on the jet deflection in both the channel and the window. Assuming that the jet travels with the same deflection from $x_{\mathrm{j}} / h=2.0$ to $l / h$, the actual jet offsets to the edge, $y_{\mathrm{a}, \mathrm{e}}$, for $\theta_{\mathrm{j}}=39^{\circ}, 50^{\circ}$, and $65^{\circ}$ were 


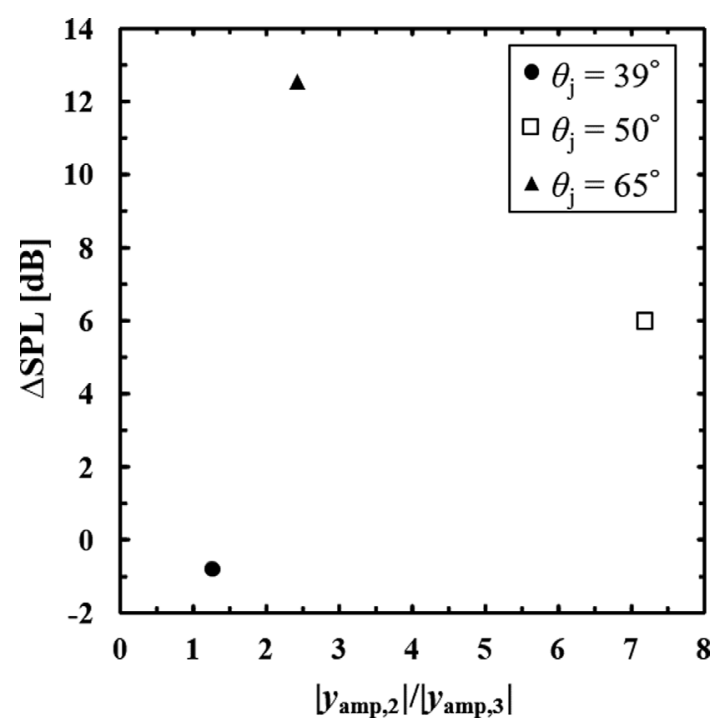

Figure 19. Relation between relative jet amplitude of second to third mode at $x_{\mathrm{j}} / h=5$ and differential SPL of second to third harmonic. $\left(y_{\mathrm{j}, \mathrm{e}}=0, l / h=5.6, Q=16 \mathrm{~L} / \mathrm{min}\right)$.

estimated to be $y_{\mathrm{a}, \mathrm{e}} / h=0.1,-0.5$, and -0.7 , respectively. This indicates that the jet deflects more inside with increasing jet angle. The absolute values of actual jet offset, $\left|y_{\mathrm{a}, \mathrm{e}} / h\right|$, corresponded almost exactly to $y_{\min } / h$ of $0.0,0.5$, and 0.7. This implies that, as jet angle increases, the $y_{\min } / h$ for the radiated sound shifts outward $\left(y_{\min }>0\right)$ by the same amount that the actual jet offset in the flow field shifts inward $\left(y_{\mathrm{a}, \mathrm{e}}<0\right)$ due to the jet deflection. To produce a harmonic structure similar to that radiated at a small jet angle, the jet offset must be increased to cancel out the inward shift in the actual jet offset. This can be also observed in Figure 12, which shows that $\theta_{\mathrm{j}, \min }$ seems to become large as $y_{\mathrm{j}, \mathrm{e}} / h$ increases. This denotes that, to minimize $\Delta$ SPL at a large jet angle, $y_{\mathrm{j}, \mathrm{e}} / h$ must be increased.

From here, the variation of SPLs in Figure 11d can be discussed. In Figure 11d, the jet offset was fixed to $y_{\mathrm{j}, \mathrm{e}} / h=0.24$; thus, the actual jet offset can be outer side by $\Delta y_{\mathrm{j}, \mathrm{e}} / h=0.24$ than that in Figure 18 under $y_{\mathrm{j}, \mathrm{e}} /$ $h=0.0$. The actual jet offset in the flow field can be $y_{\mathrm{a}, \mathrm{e}} /$ $h=0.34$ and -0.26 at $\theta_{\mathrm{j}}=39^{\circ}$ and $50^{\circ}$, respectively. At $\theta_{\mathrm{j}}=45^{\circ}, y_{\mathrm{a}, \mathrm{e}} / h$ can be around zero; therefore, $\mathrm{SPL}_{2}$ seems to exhibit its minimum at $\theta_{\mathrm{j}}=45^{\circ}$. In $\theta_{\mathrm{j}}>45^{\circ}$, the actual jet offset can be $y_{\mathrm{a}, \mathrm{e}} / h<0$, where $\mathrm{SPL}_{3}$ remains almost constant as shown in Figure 11a. Figure 14b showed that the velocity profile near the edge $\left(x_{\mathrm{j}} / h=5.0\right)$ is asymmetric unlike the assumption of the calculation [15]. Probably because of this, $\mathrm{SPL}_{3}$ varies less in $y_{\mathrm{a}, \mathrm{e}} / h<0$, and $\mathrm{SPL}_{3}$ varies little with the jet angle in the practical range.

The other factor that could be related to the SPLs of the harmonics is the jet amplitude of each mode. Figure 19 shows the relation between the relative jet amplitude of the second to third mode at $x_{\mathrm{j}} / h=5,\left|y_{\mathrm{amp}, 2}\right| /\left|y_{\mathrm{amp}, 3}\right|$, and $\triangle \mathrm{SPL}$. The jet amplitudes of the second and third modes show little correlation with the SPLs of the second and third harmonics. The variation in $\triangle \mathrm{SPL}$ with jet angle seems to be mainly caused by the inward shift of the actual jet offset due to the jet deflection.

As jet angle increases, the cavity exit moves further from the window opening, and the jet is emitted more parallel to the inner wall of the edge, as shown in Figure 9b. Further investigations into the flow field are necessary to determine how the geometrical relationships between the jet, acoustic field, and edge affect the jet deflection. There may be a possibility that the jet deflection is also caused by another blowing conditions. As shown in Figure 11c, the local variation of $\mathrm{SPL}_{2}$ around $Q=19 \mathrm{~L} / \mathrm{min}$ was observed, and it does not seem to be caused by the phase relation or the kinetic energy. It may be effective to investigate if the jet may also be deflected with changing flow rate, which can vary the SPLs.

\section{Conclusion}

This study investigated the variation of harmonic structure with jet angle in the practical range, in comparison with jet offset, flow rate, and lip-to-edge distance. Harmonic structure was evaluated by the differential sound pressure level of the second to third harmonic ( $\Delta \mathrm{SPL})$. The harmonic structure varied markedly with jet offset $(\Delta \mathrm{SPL} \approx 30 \mathrm{~dB})$, almost equally with jet angle and flow rate $(\Delta \mathrm{SPL} \approx 10$ $\mathrm{dB})$, but comparatively less with lip-to-edge distance $(\Delta \mathrm{SPL} \approx 5 \mathrm{~dB})$. The effect of jet angle on timbre seems to be an important consideration for flute players.

The mechanism for the effect of jet angle on harmonic structure was investigated based on both the radiated sound and the flow field. The radiated sound showed that, with increasing jet angle, the jet offset minimizing $\Delta \mathrm{SPL}$ increased. This denotes that there is a dependency between jet angle and jet offset. This dependency was investigated in terms of the flow field. The spatial distribution of timeaveraged jet fluctuation centers showed that the jet deflects inside with increasing jet angle. The actual jet offset estimated from the distribution was found to shift inside with increasing jet angle almost by the same amount that the jet offset minimizing $\Delta \mathrm{SPL}$ increased. This indicates that, to produce a harmonic structure similar to that radiated at a small jet angle, jet offset needs to be increased (the jet needs to be directed to the outside the edge) to cancel out the inward shift in the actual jet offset due to the jet deflection.

Other factors that could affect radiated sound were also investigated. Periodical jet fluctuations were determined by phase-averaging the measured values obtained with a hotwire anemometer. The estimated convection velocities of jet fluctuations were confirmed to be consistent with the velocity profiles. The phase relation between the jet fluctuations at the edge and the pressure fluctuations in the instrument, which is related to the condition for the acoustic power generation, seemed not to be affected significantly by jet angle. The variation in jet amplitude with jet angle showed little correlation to the variation in harmonic structure. These results support that the variation in $\triangle \mathrm{SPL}$ with 
jet angle is mainly caused by the change in the actual jet offset.

As jet angle increases, the cavity exit moves further from the window opening, and the jet is emitted more parallel to the inner wall of the edge. Further investigations into the flow field are necessary to determine how the geometrical relationships between the jet, the acoustic field, and the edge affect the jet deflection. In addition, lip shapes also depend on jet angle when a human plays the flute. For experimental results to be applicable to actual performances, it is necessary to investigate whether harmonic structure can be controlled more effectively by adjusting jet angle or lip shapes.

\section{Acknowledgments}

We would like to thank A. Natsubori for her collaboration on experiments. We also thank K. Arimoto for useful discussions. The artificial blowing device used in this study is provided by YAMAHA Corporation.

\section{Conflict of interest}

Author declared no conflict of interests.

\section{References}

1. A. Michalke: On spatially growing disturbances in an inviscid shear layer. The Journal of Fluid Mechanics 23 (1965) 521-544.

2. P. Freymuth: On transition in a separated laminar boundary layer. The Journal of Fluid Mechanics 25 (1966) 683-704.

3. J.W. Coltman: Sounding mechanism of the flute and organ pipe. The Journal of the Acoustical Society of America 44 (1968) 983-992.

4. N.H. Fletcher: Sound production by organ flue pipes. The Journal of the Acoustical Society of America 60 (1976) 926-936.

5. N.H. Fletcher, S. Thwaites: Wave propagation on an acoustically perturbed jet. Acta Acustica United With Acustica 42 (1979) 323-334.

6. S. Yoshikawa, H. Tashiro, Y. Sakamoto: Experimental examination of vortex-sound generation in an organ pipe: A proposal of jet vortex-layer formation model. The Journal of Sound and Vibration 331 (2012) 2558-2577.

7. N.H. Fletcher, L.M. Douglas: Harmonic generation in organ pipes, recorders, and flutes. The Journal of the Acoustical Society of America 68 (1980) 767-771.

8. R. Auvray, A. Ernoult, B. Fabre: Time-domain simulation of flute-like instruments: Comparison of jet-drive and discretevortex models. The Journal of the Acoustical Society of America 136 (2014) 389-400.

9. A.H. Benade: Fundamentals of Musical Acoustics, second, revised edition. Dover Publications, 1990.

10. B. Fabre, J. Gilbert, A. Hirschberg, X. Pelorson: Aeroacoustics of musical instruments. Annual Review of Fluid Mechanics 44 (2012) 1-25.

11. Y. Ando: Drive conditions of a flute and their influences upon sound pressure level and fundamental frequency of generated tone: An experimental study of a flute I. The Journal of the Acoustical Society of Japan 26 (1970) 253-260.
12. Y. Ando: Drive conditions of a flute and their influences upon harmonic structure of generated tone: An experimental study of a flute II. The Journal of the Acoustical Society of Japan 26 (1970) 297-305.

13. P. de la Cuadra: The Sound of Oscillating Air Jets: Physics, Modeling and Simulation in Flute-like Instruments. PhD thesis, Stanford University, 2005.

14. C. Vauthrin, B. Fabre, I. Cossette: How does a flute player adapt his breathing and playing to musical tasks? Acta Acustica United With Acustica 101 (2015) 224-237.

15. S. Yoshikawa: Harmonic generation mechanism in organ pipes. The Journal of the Acoustical Society of Japan (E) 5 (1984) $17-29$.

16. J.W. Coltman: Jet offset, harmonic content, and warble in the flute. The Journal of the Acoustical Society of America 120 (2006) 2312-2319.

17. M.P. Verge, B. Fabre, W.E.A. Mahu, A. Hirschberg, R.R. van Hassel, A.P.J. Wijnands: Jet formation and jet velocity fluctuations in a flue organ pipe. The Journal of the Acoustical Society of America 95 (1994) 1119-1132.

18. R. Auvray, B. Fabre: Specific features of a stopped pipe blown by a turbulent jet: Aeroacoustics of the panpipes. The Journal of the Acoustical Society of America 139 (2016) $3214-3225$

19. K. Onogi, H. Yokoyama, A. Iida: Analysis of jet oscillations with acoustic radiation in the recorder by direct aeroacoustic simulations. The Journal of the Acoustical Society of America 146 (2019) 1427-1437.

20. N.H. Fletcher, T.D. Rossing: The Physics of Musical Instruments. 2nd ed. Springer Verlag, 1998.

21. J.W. Coltman: Jet drive mechanism in edge tones and organ pipes. The Journal of the Acoustical Society of America 60 (1976) 725-733.

22. S.A. Elder: On the mechanism of sound production in organ pipes. The Journal of the Acoustical Society of America 54 (1973) 1554-1564.

23. N.H. Fletcher: Jet drive mechanism in organ pipes. The Journal of the Acoustical Society of America 60 (1976) 481-483.

24. L. Cremer, H. Ising: Die selbsterregten Schwingungen von Orgelpfeifen (The self-excited oscillation of organ pipes). Acta Acustica United With Acustica 19 (1967) 143-153.

25. S. Yoshikawa, J. Saneyoshi: Feedback excitation mechanism in organ pipes. The Journal of the Acoustical Society of Japan 1 (1980) 175-191.

26. Y. Sawada, S. Sakaba: On the transition between the sounding modes of a flute. The Journal of the Acoustical Society of America 67 (1980) 1790-1794.

27. A. Ernoult, P. de la Cuadra, B. Fabre: An inclined plane: A simple model for the acoustic influence of the flutist's face. Acta Acustica United With Acustica 104 (2018) 496-508.

28. K. Arimoto: Experimental study on the temporal fluctuation of harmonics in flute sounds, in Proc. International Symposium on Music Acoustics, 13-17 September, Detmold, Germany. 2019.

29. T. Matsuno, S. Honami, K. Fujii, S. Sekimoto, A. Iida: Flow measurement technique. The Journal of Plasma and Fusion Research 91 (2015) 661-664.

30.S. Yoshikawa: Jet-wave amplification in organ pipes. The Journal of the Acoustical Society of America 103 (1998) 2706-2717.

31. P. de la Cuadra, C. Vergez, B. Fabre: Visualization and analysis of jet oscillation under transverse acoustic perturbation. The Journal of Flow Visualization and Image Processing 14 (2007) 355-374. 


\section{Appendix}

The variations of harmonic structure with jet angle were also measured for a flute with a body and a foot joint. The measurements were performed with the fingering of A4. The lip-to-edge distance was fixed to the actual blowing condition shown in Table $1(l / h=5.6)$. The flow rate was fixed to $Q=10 \mathrm{~L} / \mathrm{min}\left(U_{0}=13.4 \mathrm{~m} / \mathrm{s}\right)$, where the fundamental frequencies of radiated sounds were almost $440 \mathrm{~Hz}$. Figure A.1 shows the variations of the SPLs measured by fixing jet offsets to values within the practical range. The jet angle that $\triangle \mathrm{SPL}$ exhibits its minimum is $\theta_{\mathrm{j}, \min }=37^{\circ}$ for $y_{\mathrm{j}, \mathrm{e}} / h=0.17$ and $\theta_{\mathrm{j}, \min }=43^{\circ}$ for $y_{\mathrm{j}, \mathrm{e}} / h=0.24$. From this result, the actual jet offset $\left(y_{\mathrm{a}, \mathrm{e}} / h\right)$ probably changes by about -0.07 due to the jet deflection when the jet angle increases $\theta_{\mathrm{j}, \min }=37^{\circ}$ to $43^{\circ}$. Since the minimum angles

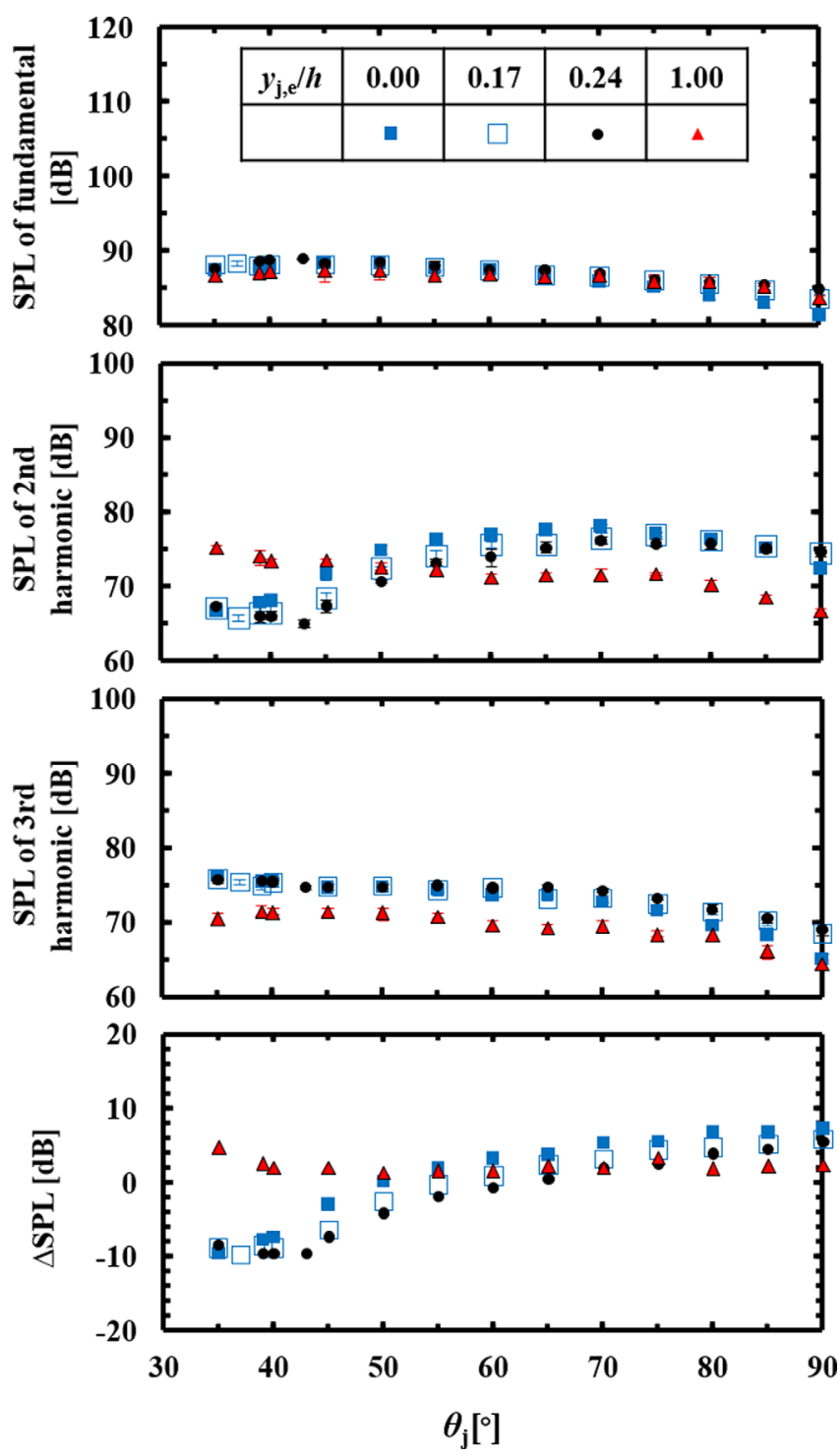

Figure A.1. Variations of harmonic structure with jet angle measured for flute with A4 fingering. Lip-to-edge distance was fixed to the actual blowing condition shown in Table $1(l / h=5.6)$. Flow rate was fixed to $Q=10 \mathrm{~L} / \min \left(U_{0}=13.4 \mathrm{~m} / \mathrm{s}\right)$. $\theta_{\mathrm{j}, \text { min }}$ for $y_{\mathrm{j}, \mathrm{e}} / h=0.17$ and 0.24 almost correspond to $\theta_{\mathrm{j}}$ min in Figure 12 measured for the head joint, the jet deflection with jet angle is almost the same between the flute with the A4 fingering and the head joint.

In addition, the variations of harmonic structure with jet angle were investigated for the fingerings of A4, A5 $(880 \mathrm{~Hz})$, and A6 $(1760 \mathrm{~Hz})$, as shown in Figure A.2. The lip-to-edge distance and the jet offset were fixed to the actual blowing conditions shown in Table $1(l / h=5.6$, $\left.y_{\mathrm{j}, \mathrm{e}} / h=0.24\right)$. The flow rate was fixed to $Q=16 \mathrm{~L} / \mathrm{min}$ $\left(U_{0}=21.5 \mathrm{~m} / \mathrm{s}\right)$ for A5 and $Q=32 \mathrm{~L} / \mathrm{min}\left(U_{0}=43.0\right.$ $\mathrm{m} / \mathrm{s}$ ) for $\mathrm{A} 6$. The radiated sounds were investigated within the range that the fundamental frequency is \pm 10 cent of A4, A5, or A6. With increasing jet angle, the curves of

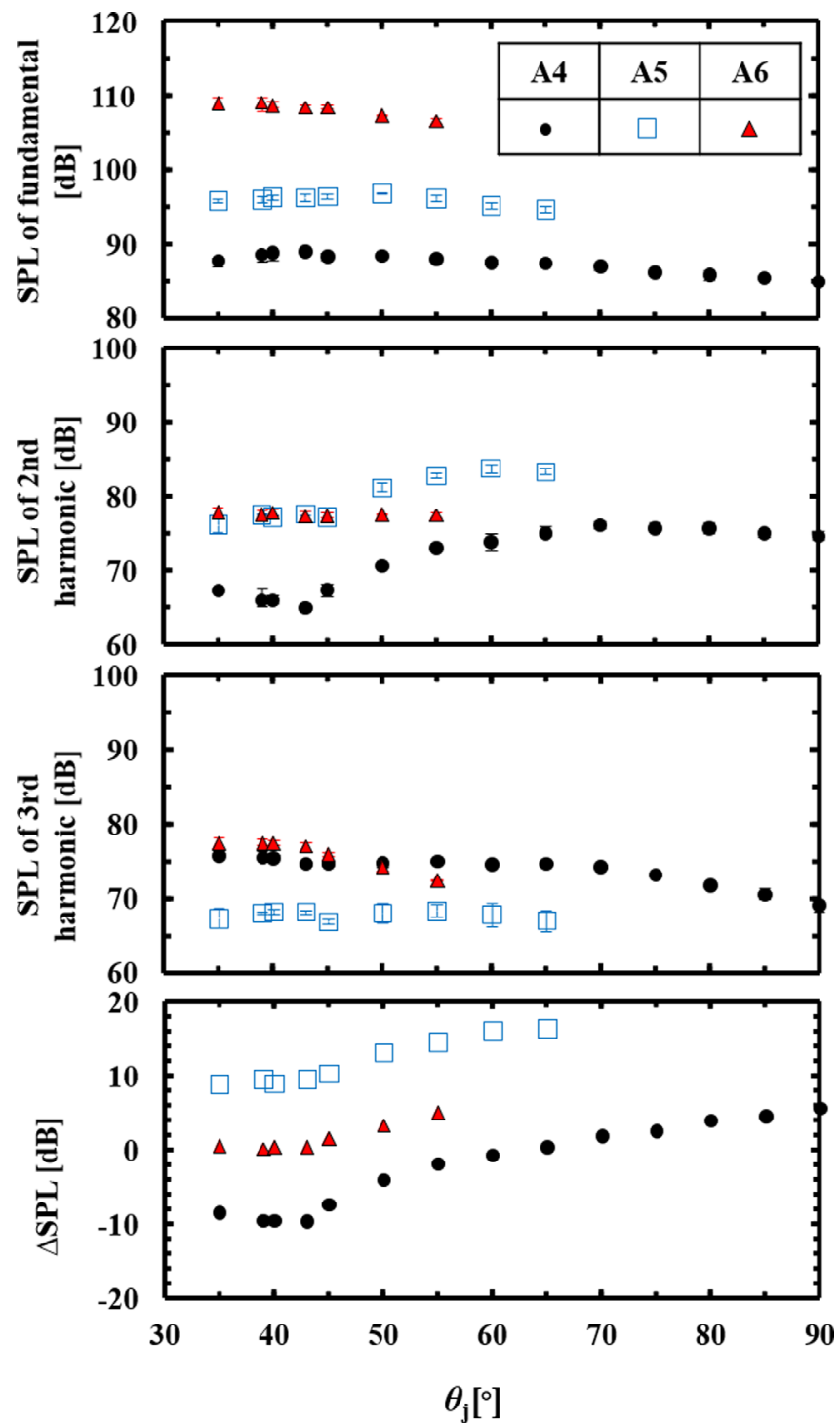

Figure A.2. Variations of harmonic structure with jet angle measured for flute with A4, A5, and A6 fingering. Lip-to-edge distance and jet offset were fixed to the actual blowing conditions shown in Table $1\left(l / h=5.6, y_{\mathrm{j}, \mathrm{e}} / h=0.24\right)$. Flow rate was fixed to $Q=16 \mathrm{~L} / \mathrm{min}\left(U_{0}=21.5 \mathrm{~m} / \mathrm{s}\right)$ for $\mathrm{A} 5$ and $Q=32 \mathrm{~L} / \min \left(U_{0}=43.0 \mathrm{~m} / \mathrm{s}\right)$ for $\mathrm{A} 6$. 
$\Delta \mathrm{SPL}$ for $\mathrm{A} 5$ and $\mathrm{A} 6$ almost remain flat in $\theta_{\mathrm{j}}<43^{\circ}$ but increase in $\theta_{\mathrm{j}}>43^{\circ}$. For A4, A5, and A6 at $y_{\mathrm{j}, \mathrm{e}} / h=0.24$, the actual jet offset seems to be zero around $\theta_{\mathrm{j}}=43^{\circ}$. The jet seems to deflect inside the edge with increasing jet angle in $\theta_{\mathrm{j}}>43^{\circ}$, which respectively promotes an increase and a decrease of the second and the third harmonic. There is no noticeable shift in the $\Delta \mathrm{SPL}$ curve with tone range.

From these results, the variations of harmonic structure with jet angle measured for the head joint can also occur over the flute's three ranges.

Cite this article as: Onogi K, Yokoyama H \& Iida A. 2021. Effects of jet angle on harmonic structure of sound radiating from the flute. Acta Acustica, $\mathbf{5}, 11$. 